\title{
Nanoscale electrochemical 3D deposition of cobalt with nanosecond voltage pulses in an STM
}

\author{
Alain Reiser ${ }^{1,2}$, Rolf Schuster ${ }^{3}$, Ralph Spolenak $^{1 *}$ \\ ${ }^{1}$ Laboratory for Nanometallurgy, Department of Materials, Federal Institute of Technology (ETH) Zürich, 8093 Zürich, Switzerland \\ ${ }^{2}$ Department of Materials Science and Engineering, Massachusetts Institute of Technology, MA-02139 Cambridge, United States of America \\ ${ }^{3}$ Institute of Physical Chemistry, Karlsruhe Institute of Technology, 76131 Karlsruhe, Germany \\ *To whom correspondence should be addressed; E-mail: ralph.spolenak@mat.ethz.ch
}

Keywords: Additive manufacturing, nanoscale, microscale, metal, electrodeposition

To explore a minimal feature size of $<100 \mathrm{~nm}$ with electrochemical additive manufacturing, we use a strategy originally applied to microscale electrochemical machining for the nanoscale deposition of Co on Au. The concept's essence is the localization of electrochemical reactions below a probe during polarization with ns-long voltage pulses. As shown, a confinement that exceeds that predicted by a simple model based on the time constant for one-dimensional double layer charging enables a feature size of $<\mathbf{5 0} \mathbf{~ n m}$ for $2 \mathrm{D}$ patterning. We further indirectly verify the potential for out-of-plane deposition by tracking growth curves of high-aspect-ratio deposits. Importantly, we report a lack of anodic stability of Au tips used for patterning. As an inert probe is the prerequisite for controlled structuring, we experimentally verify an increased resistance of $\mathrm{Pt}$ probes against degradation. Consequently, the developed setup and processes show a path towards reproducible direct 2D and 3D patterning of metals at the nanoscale.

Nanoscale additive manufacturing (AM) of inorganic materials offers exciting prospects for nanomanufacturing, enabling 3D structures for sensing ${ }^{\sqrt[112]{2}}$ or plasmonic ${ }^{3}$ applications, or additive synthesis of multi-materials with extremely high resolution ${ }^{4}$. Yet, the deposition of metals of high purity with a resolution $<100 \mathrm{~nm}$ is challenging with current AM methods ${ }^{5}$. Materials grown by focused electron/ion-beam-induced deposition (FEBID/FIBID) using conventional precursors notoriously suffer from high carbon contents ${ }^{6}$, although high metal contents have been enabled by the use of UHV systems $^{7}$ ( $>95 \% \mathrm{Fe}$ ) or carbon-free but unstable precursors like gold-trifluorophosphine-chloride ${ }^{8}$. Post-growth purification often results in shrinkage and warping of structures (chemical purification with high geometric fidelity has recently been demonstrated for needle-like structures ${ }^{3}$ ). Techniques based on dispensing of nanoparticle inks suffer from similar complications during post-print thermal annealing, with evolution of porosity and warping being a common issue $\mathrm{e}^{910}$. In addition, ink-based methods are limited to a feature size larger than a few particle diameters due to clogging issues — with smallest particle diameters of a few nanometers, minimum line widths of several tens of nanometers can probably not be expected (currently, the smallest line width of annealed inks is $80 \mathrm{~nm} \frac{11}{1}$, and direct ink writing (DIW) achieves minimal line widths of $0.6-2 \mu \mathrm{m}^{12}$ ). To avoid potentially damaging post- 
deposition procedures, direct metal deposition is advantageous. However, not many direct-deposition techniques can possibly reach the length scale in question. The low transfer accuracy of laser-induced forward transfer (LIFT) renders advances towards nanoscale patterning extremely challenging. As much needed alternatives, electrochemical methods - deposition of pure metals from particle-free salt solutions, using nanometer probes to guarantee accuracy and resolution - may offer a pathway to nanoscale 3D deposition of high-purity metals with access to the $10 \mathrm{~nm}$-regime. Indeed,recent developments in electrochemical AM methods demonstrate a feature size smaller than $100 \mathrm{~nm} \frac{413}{4}$.

To evaluate options for further decreasing the minimal feature size of electrochemical concepts, it is helpful to consider the current limitations of state-of-the-art techniques for 2D and 3D patterning, which include nozzle-based methods (all current AM concepts) and tip-based methods (up to date used for $2 \mathrm{D}$ patterning only). The nozzle-based concepts have shown smallest 3D feature size of $\geq 25 \mathrm{~nm}$ (meniscus-confined electrodeposition, MCED) and $\approx 100 \mathrm{~nm}^{4}$ (electrohydrodynamic redox printing, EHD-RP). In first approximation, the feature size of MCED is equal to the diameter of the capillary. The minimal feature size to be achieved with current feedback principles is estimated to be $25 \mathrm{~nm}$, with smaller feature size prohibited by experimentally challenging prevention of nozzle clogging 13 . In EHD-RP, a mere decrease of nozzle aperture from 160 to $100 \mathrm{~nm}$ did not result in a decreased but rather increased feature size ${ }^{4}$. A third approach, concentration-confined electrodeposition $^{14}$, is presently unsuitable for nanoscale deposition due to the poor confinement of deposition - the use of apertures as small as 30-50 nm merely decreased the feature size to $400-600 \mathrm{~nm} \frac{15}{}$.

In contrast, electrochemical 2D patterning with nanoscale-sharp tips (as those typically used in scanning probe microscopy, SPM) enables a feature size close to $10 \mathrm{~nm}$ - the smallest feature size demonstrated was $16 \mathrm{~nm}$ for Co deposits $\frac{16}{16}$ and $\approx 10 \mathrm{~nm}$ for $\mathrm{Cu}^{17}$. Importantly, the absence of a clogging issue with scanning probes guarantees minimal dimensions of the structuring tools and thus the potential for smallest feature sizes. However, the localization of electrodeposition and the supply of ions is less straightforward than with capillary-based printing nozzles. Of the methods suggested in literature, dip-pen lithography ${ }^{18}$ is the only air-based technique, supplying ions through a liquid meniscus between substrate and an atomic force microscope (AFM) tip (similar to the meniscus used in MCED). Localization of deposition in the meniscus is straightforward, but the limited reservoir of ions (metal salts dried on the AFM tip) is not ideal for continuous deposition. As a consequence, the majority of proposed techniques relies on the supply of ions from a bath of electrolyte solution. Immersing the probe and the substrate into a solution of metal salts guarantees a practically infinite supply of cations for electrodeposition, but demands more advanced strategies for localization of the electrodeposition. The proposed concepts for localized growth below a SPM tip immersed in an electrolyte bath can be grouped into two fundamental categories: first, the limitation of the active surface area, and second, a local modification of the factors that govern either the current distribution, the kinetics or the energetics of the electrochemcial reaction. The first group is not suited for 3D deposition, as it merely relies on the local activation of a generally passivated substrate ${ }^{19}$ _ an approach that is limited to deposition directly on the substrate and offers no means for out-of-plane growth. In the second group, methods that utilize the inhomogeneous current distribution below a tip suffer from low resolution (a minimal feature size of $2 \mu \mathrm{m}$ was achieved by using the tip as a local anode upon 
direct current electrodeposition $\underline{20}$ ), and methods that locally introduce surface defects for preferential nucleation ${ }^{21 / 22}$ show an inability for $3 \mathrm{D}$ deposition. The only concepts that guarantee strict localization of the deposition (and thus high resolution) in combination with no need for modifications of the substrate (and thus the possibility to enter the third dimension) are those that energetically limit the spatial extent of cathodic reactions. Energetic localization of electrochemical deposition is based on a local increase of the activation or concentration overpotential. The approach guarantees strong confinement due to the exponential relationship between growth rate and overpotential. Two concepts that make use of the exponential confinement have demonstrated deposition of metals with nanometric resolution. The first is based on the manipulation of the local Nernst potential $\frac{16}{16}$, and the second relies on spatially containing the charging of the cathode's double-layer capacitance by nanosecond voltage pulses 23 . While we have experimentally studied both strategies, we have more successfully implemented the second approach and thus think it is a more robust pathway towards 3D deposition with an electrochemical STM - probably due to its relaxed requirements to the stability of the substrate's potential (approximately $\pm 100 \mathrm{mV}$ versus $20 \mathrm{mV}$ ) and the possibility to use electrolytes of high concentration ( $1 \mathrm{M}$ versus $1 \mathrm{mM})$.

The concept of confinement by spatial limitation of double-layer charging is illustrated best with the simplified equivalent circuits for two current paths (1) and (2) that connect tip and substrate in Figure 19. Typically, the surface potential of a conductive electrode immersed in an electrolyte solution, that is, the voltage drop across its electrochemical double layer (DL), is constant across a well conductive electrode's expanse. Yet, the application of nanosecond voltage pulses between the tip and the substrate results in an effective confinement of current flow through path (1) and hence a localization of double-layer polarization and all electrochemical reactions to regions connected by this path. The confinement originates from varying time constants $\tau=R C=\rho d \cdot c_{\mathrm{DL}}$ that describe the transient voltage $U_{\mathrm{C}}(t)$ for each of the current paths upon pulsing the potential $U$ applied between tip and substrate. As the double layer capacitance per area $c_{\mathrm{DL}}$ and the specific electrolyte resistance $\rho$ can be assumed to be constant, $\tau(d)$ is directly proportional to the local distance $d$ to the tip and $\tau_{1}<\tau_{2}$. Additionally, $U_{\mathrm{C}}(t)$ becomes a function of not only $t$ but also $d$. During a voltage pulse of duration $t_{\mathrm{p}}$, the local polarization $U_{\mathrm{C}}(d)$ reaches significant values only if the local $\tau(d)$ is on the order of the pulse width $t_{\mathrm{p}}$, strongly confining electrochemical reactions to areas close to the tip (and making the amount of confinement proportional to the pulse length). A pulse width of $30 \mathrm{~ns}$ is estimated to limit polarization within a distance $d$ of approximately $1 \mu \mathrm{m}$ in a typical aqueous electrolyte solution $\left(0.1 \mathrm{M} \mathrm{HClO}_{4}\right)^{\sqrt{23}}$, or $d=200 \mathrm{~nm}$ in a non-aqueous solution with lower conductivity $(1 \mathrm{M} \mathrm{LiCl} \text { in DMSO })^{24}$. The concept has mainly been used for micromachining of various metals and p-Si with minimal gap widths between tool and trench down to $20 \mathrm{~nm}^{23 / 25 / 26}$. Demonstrations of the reverse process - deposition of micrometer-sized $\mathrm{Cu}$-islands (using a micrometer-sized tool) ${ }^{23}$ and $\approx 10 \mathrm{~nm}$-wide $\mathrm{Cu}$ dots (with an STM tip) 17 _ showed a potential for unrivaled minimal feature sizes in electrochemical AM. Yet, no 3D deposition has been reported so far, and even the mentioned reports of 2D deposition have a character of preliminary results.

Here, we explore possibilities for nanoscale 3D deposition with nanosecond voltage pulses, motivated by the fact that this approach has shown smallest 2D feature sizes amongst all electrochemical 

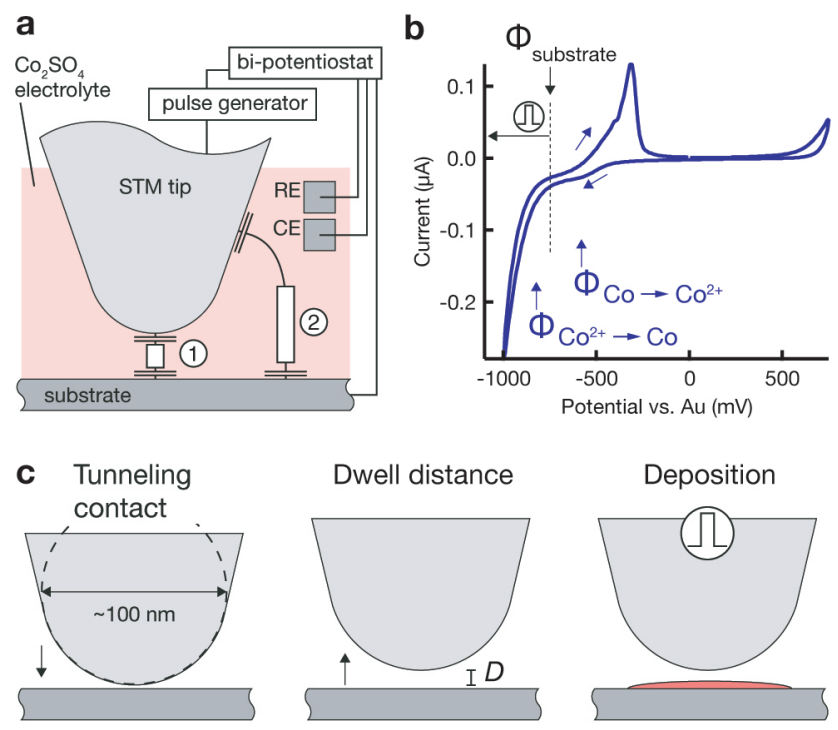

Deposition
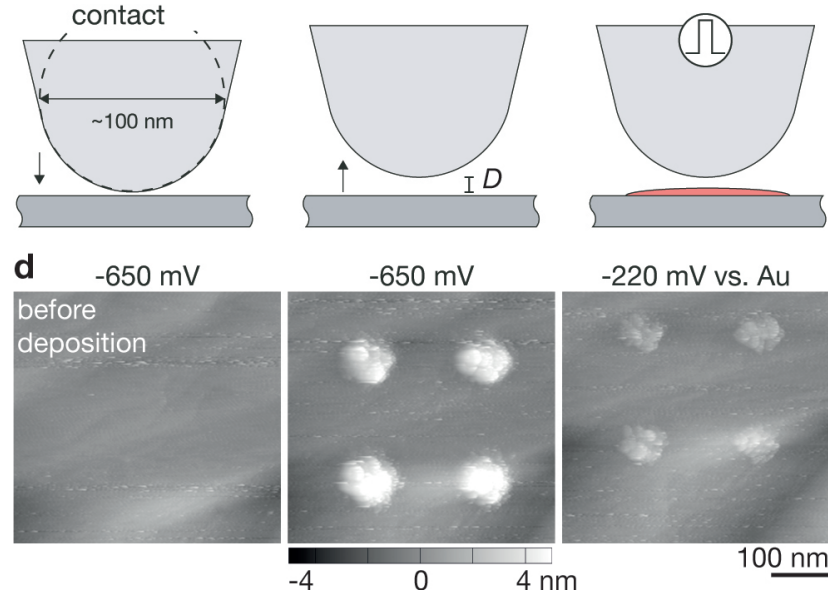

$-220 \mathrm{mV}$ vs. Au

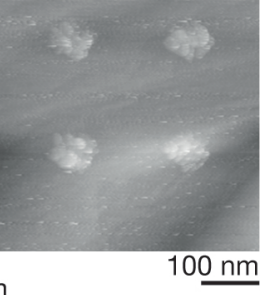

Figure 1: Localized Co deposition by nanosecond voltage pulses. a, Schematic of the experimental setup for localized electrochemical deposition. Current paths (1) and (2) are representative of paths with low (1) and high (2) time constant for DL charging $\tau=R C=\rho d \cdot c_{\mathrm{DL}}$. Application of nanosecond voltage pulses limits charging of the substrate's DL to regions connected by current path (1). b, Cyclic voltammogram of a Au substrate in a solution of $1 \mathrm{M} \mathrm{CoSO}_{4}$ in DMSO. During and after deposition, the substrate potential $\Phi_{\text {substrate }}$ is potentiostatically set to $\Phi_{\mathrm{Co}^{2+} \rightarrow \mathrm{Co}}<\Phi_{\text {substrate }}<\Phi_{\mathrm{Co} \rightarrow \mathrm{Co}^{2+}}$, preventing delocalized deposition of Co as well as dissolution of local Co deposits. Upon application of positive nanosecond voltage pulses to the tip, the substrate is locally polarized towards more negative potentials, initiating local Co deposition. c, Schematic of the deposition procedure (drawn to scale). Tip and substrate are immersed in a $\mathrm{Co}^{2+}$-containing electrolyte solution. The tip is positioned at a defined dwell distance $d$ from the substrate by approach of the tip until tunneling contact and subsequent retraction by a distance $d$ (typically, $d=10 \mathrm{~nm}$ ). Application of nanosecond voltage pulses (10-100 ns) to the tip locally polarizes the substrate below the tip and initiates deposition. d, Deposition is verified by pre- and post-deposition scans of the substrate. STM scans of a Au surface before and after four deposition events ( $1 \mathrm{M} \mathrm{CoSO}_{4}$ in $\left.\mathrm{DMSO}\right)$, and after increasing the surface potential to a value $\Phi_{\text {substrate }}>\Phi_{\mathrm{Co} \rightarrow \mathrm{Co}^{2+}}$. The incomplete dissolution suggests that the deposits were mostly Co but contained Au contamination due to a degrading tip. Surface potentials (vs. Au) are stated above the images. Deposition parameters. Electrolyte: $1 \mathrm{M} \mathrm{CoSO}_{4}$ in DMSO; pulse length: $100 \mathrm{~ns}$; pulse amplitude: $6 \mathrm{~V}$; duty cycle: 0.01; pulses per point: 3000; dwell distance: $10 \mathrm{~nm}$; tip: Au. 
AM techniques. We demonstrate reproducible 2D patterning and out-of-plane growth using nanosecond voltage pulses to localize the deposition in an electrochemical STM. Utilizing STM probes as tools for local deposition, we demonstrate sub-100 nm 2D structuring of Co on Au in a DMSO electrolyte. Importantly, we find that the achieved feature size is not explained by the limited spatial extent of charging of the double layer alone, and additional kinetic or energetic mechanisms must be responsible for the localization of deposition. We further discuss challenges for 3D deposition and reproducibility (in aqueous and non-aqueous solvents), both related to the electrochemical stability of the used metallic STM tips. As a result, we show improved inertness of Pt or Pt-20at.\%Ir probes in aqueous electrolytes. With improved reproducibility and proof-of-concept demonstrated here, future exploration of the method for direct out-of-plane deposition offers the potential for a simple, economic alternative to FEBID for applications that need high purity of metals.

\section{Results and discussion}

\section{The deposition procedure}

Figure 1 sketches the basic procedure for localized deposition of Co on a Au substrate utilizing a Au STM tip as a tool for initiating deposition. First, it is important to note that the surface potentials of the two working electrodes, $\Phi_{\text {tip }}$ and $\Phi_{\text {substrate, }}$ are continuously controlled by a low-frequency bi-potentiostat (versus a Au quasi-reference electrode, RE). Both electrodes are held at a potential that avoids any faradaic reactions related to the deposition or dissolution of Co. Typically, $\Phi_{\text {tip }}$ and $\Phi_{\text {substrate }}$ are selected to be in-between the onset potential of Co deposition $\Phi_{\mathrm{Co}^{2+} \rightarrow \mathrm{Co}}$ and Co dissolution $\Phi_{\mathrm{Co} \rightarrow \mathrm{Co}^{2+}}$, as indicated in the cyclic voltammogram of the Au substrate in Figure $1 \mathrm{p}$ ( $\Phi_{\text {tip }}$ is $\approx 200 \mathrm{mV}$ positive to $\left.\Phi_{\text {substrate }}\right)$. The rationale for these potentials is simple: $\Phi<\Phi_{\mathrm{Co}^{2+} \rightarrow \mathrm{Co}}$ would result in continuous, non-localized electrodeposition of Co onto the substrate (or the tip); in contrast, $\Phi>$ $\Phi_{\mathrm{Co} \rightarrow \mathrm{Co}^{2+}}$ would lead to the immediate dissolution of any localized deposit.

Prior to any deposition, an image of the substrate is recorded by scanning of the surface with the setup in feedback mode. For deposition, the tip is positioned 10-100 nm above the substrate by first approaching the surface until a tunneling current is detected (defined as "tunneling contact") and then retracting the tip by the respective distance $d$ into its dwell position (Figure 1 ;). In this position, the setup is switched into pulse mode, i.e., the STM tip is connected to the output of the pulse generator. For deposition, a pulse train of a predefined number of pulses (typically 100-100 000 pulses of $10-100 \mathrm{~ns}$ at a duty cycle of $0.01-0.1$ and an amplitude of $+1-6 \mathrm{~V}$ ) is applied to the tip. As described in detail elsewhere ${ }^{23 / 25 / 27}$, the high-frequency pulses are added to the baseline potential of the tip $\Phi_{\text {tip }}$ (and with opposite polarity to $\Phi_{\text {substrate }}$ ), which results in a local decrease of the surface potential $\Phi_{\text {substrate }}$ to values $<\Phi_{\mathrm{Co}^{2+} \rightarrow \mathrm{Co}}$ and initiates local growth. After deposition, the system is returned into feedback mode and the deposits are imaged. To judge whether the deposited material is Co, the surface potential is lifted to values $\Phi_{\text {substrate }}>\Phi_{\mathrm{Co} \rightarrow \mathrm{Co}^{2+}}$. STM scans documenting such a depositiondissolution cycle are shown in Figure $1 \mathrm{~d}$. The dissolution of large fractions of the deposited material at $\Phi_{\text {substrate }}>\approx 400 \mathrm{mV}$ matches the behavior of Co predicted by the cyclic voltammogram in Figure $1 \mathrm{~b}$ and strongly suggests that the major constituent of the deposits is Co. Indeed, the deposition of 
any element other than Co from the used electrolyte solutions is unlikely. The high purity of the Co salts $(99.999 \%)$ and the solvents used, the harsh cleaning of the electrochemical cell and its components, in combination with the high concentration of $\mathrm{Co}^{2+}$ ions in the solution render the deposition of mere contamination unlikely. Nonetheless, the deposition of $\mathrm{Co}$ is still an assumption that should be confirmed with a standard chemical analysis technique ex situ once larger structures can be printed.

Incomplete dissolution of deposits in Figure $1 \mathrm{~d}$ at a potential $\Phi_{\mathrm{Co} \rightarrow \mathrm{Co}^{2+}}<\Phi_{\text {substrate }}<\Phi_{\mathrm{Au} \rightarrow \mathrm{Au}^{3+}}$ indicates unwanted contamination due a degradation of the Au tip when used as the anode during voltage pulsing. Supplementary section 2.1 directly confirms loss of Au tip material during deposition experiments and also deposition of material below an Au tip in inert electrolytes with typical pulse parameters. We thus conclude that tip material is likely dissolved as anodic reaction during voltage pulsing and a fraction of $\mathrm{Au}$ is co-deposited with $\mathrm{Co}$, causing the observed incomplete dissolution of deposits. However, it is unclear whether removal of tip material can be attributed to oxidation alone, as mere field instabilities during high voltage pulses are also known to destroy STM probes. In any case, the pronounced anodic instability of Au under typical deposition conditions necessitates an alternative tip material for future applications. While most of the presented results were obtained with Au tips, we have identified Pt and Pt-Ir tips as more stable tip materials. Supplementary Section 2.2 presents much-improved anodic stability of Pt and Pt-Ir tips sufficient for deposition of Co deposits that show complete dissolution (Supplementary Figure S8). Hence, the deposition of presumably pure Co structures is enabled with Pt and Pt-20at.\%Ir tips and there is potential for stable and controlled deposition without tip degradation.

\section{D patterning}

2D patterns are generated with the point-by-point deposition approach previously described. A spacing of $100 \mathrm{~nm}$ between individual deposition events results in patterns of discrete dots (Figure $2 \mathrm{a}$ ), while a smaller spacing typically leads to a seemingly continuous film (b) or lines (d) (See Supplementary Figure S3 for an SEM micrograph of a grid). Note the strong spatial confinement of the grown patterns - two atomically thin terrace edges of the (111) Au substrate are still visible in closest proximity to the deposited film in Figure $2 \mathrm{~b}$ (arrow). In general, all deposited structures are very much two-dimensional. Typical out-of-plane aspect ratios are $\ll 1$, for example $\approx 0.1$ for the line in Figure $2 \mathrm{~d}$.

In contrast to the point-wise deposition strategy, continuous translation of the tip at a fixed distance to the substrate and simultaneous polarization with nanosecond voltage pulses has not resulted in reproducible deposition up to date. 
a

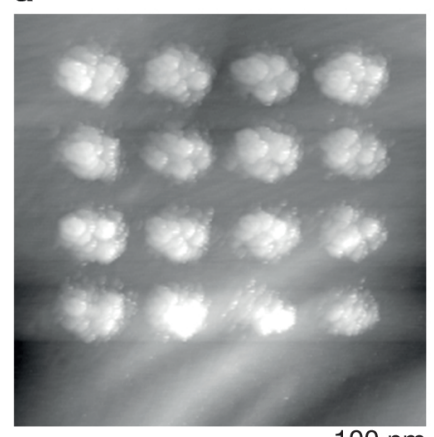

$100 \mathrm{~nm}$

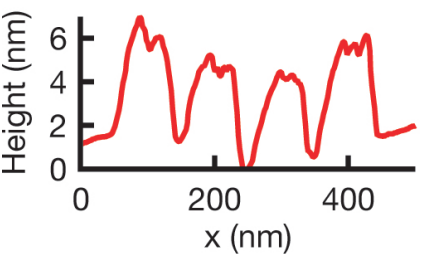

b

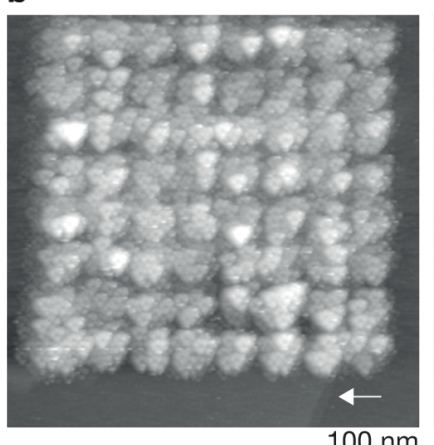

$100 \mathrm{~nm}$

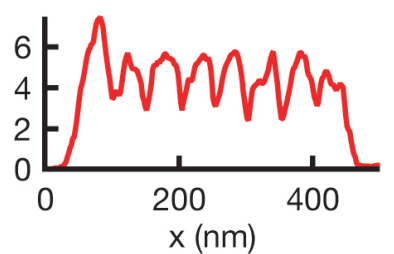

C

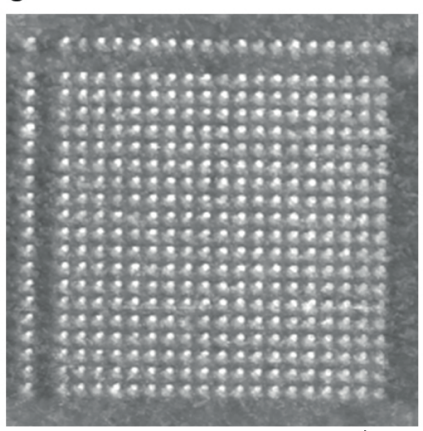

$1 \mu \mathrm{m}$

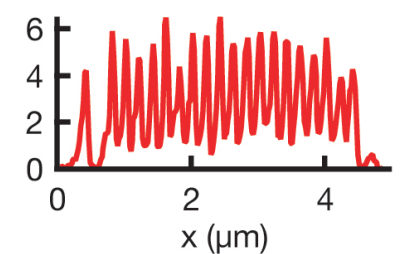

d

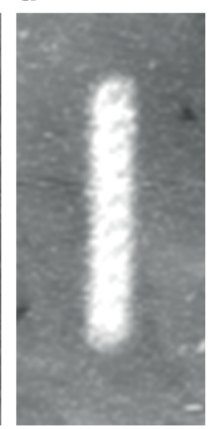

$200 \mathrm{~nm}$

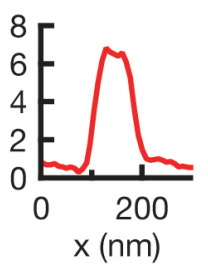

e

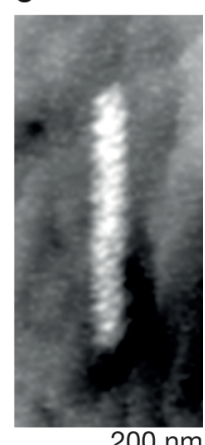

$200 \mathrm{~nm}$

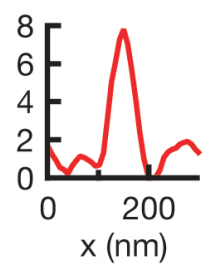

Figure 2: 2D patterning. STM scans of patterns grown with a point-by-point strategy. Point-to-point spacing: a, $100 \mathrm{~nm}, \mathbf{b}, 50 \mathrm{~nm}, \mathbf{c}, 200 \mathrm{~nm}, \mathbf{d}, \mathbf{e}, 50 \mathrm{~nm}$. The arrow in (b) points at two terrace edges of the (111) Au substrate - their visibility illustrates the strong confinement of the Co deposition.

Deposition parameters. Electrolyte: $1 \mathrm{M}(\mathrm{a}-\mathrm{c}), 0.5 \mathrm{M}(\mathrm{d}, \mathrm{e}) \mathrm{CoSO}_{4}$ in DMSO; pulse length: $100 \mathrm{~ns}$; pulse amplitude: $6 \mathrm{~V}(\mathrm{a}-\mathrm{c}), 5 \mathrm{~V}(\mathrm{~d}, \mathrm{e})$; duty cycle: 0.01 ; pulses per point: $3000(\mathrm{a}-\mathrm{c}), 2000(\mathrm{~d}, \mathrm{e})$; dwell distance: $10 \mathrm{~nm}(\mathrm{a}-\mathrm{d}), 30 \mathrm{~nm}(\mathrm{e})$.

\section{Out-of-plane deposition}

In general, more pulses per deposition event increase the height of the deposit (Supplementary Figure S4). Yet, the height of a dot grown upon a single deposition event, i.e., when firing a defined number of pulses while the tip is in its dwell position, must be smaller than the dwell distance. Otherwise, the deposit will short-circuit the tip and substrate, which usually results in large and poorly confined deposits. Hence, for a typical dwell distance of $10 \mathrm{~nm}$ and a lateral feature size of $50-100 \mathrm{~nm}$, the maximum aspect ratio of a single deposit is 0.1-0.2. Building out-of-plane structures thus requires the sequential "stacking" of individual deposits. The respective procedure is outlined in Figure 3 a. After each dwell, the tip approaches the surface, detects the out-of-plane position of the grown deposit, and retracts by the defined dwell distance $d$. This routine maintains a constant spacing between tip and growing structure. The out-of-plane growth of the deposit can be tracked by recording the $z$-position of contact between tip and deposit. Graphs that plot this data are thus termed "growth curves".

Figure $3 \mathrm{~b}$ presents 3D array of dots deposited by increasing the number of deposition events from left to right. Note that the width of the deposits does not increase with height (except after the first deposition event). The corresponding cross-section line profile demonstrates the gradual increase in height of the individual dots (c), as is also indicated by the corresponding growth curves in panel (d). Thus, out-of-plane growth is in principle accessible and can be tracked with the growth curves. Growth curves for larger numbers of consecutive deposits suggest an out-of-plane deposition rate of up to $5 \mathrm{~nm} \mathrm{~s}^{-1}$ in DMSO (Figure 3e, 3.5 V) and $20 \mathrm{~nm} \mathrm{~s}^{-1}$ in water (Figure 3f).

The irregular progress of growth indicates instabilities in the process (Figure 3 e). For a pulse amplitude of $2 \mathrm{~V}$, no growth occurs. Increasing the pulse amplitude to 3-4 V results first in a dip 

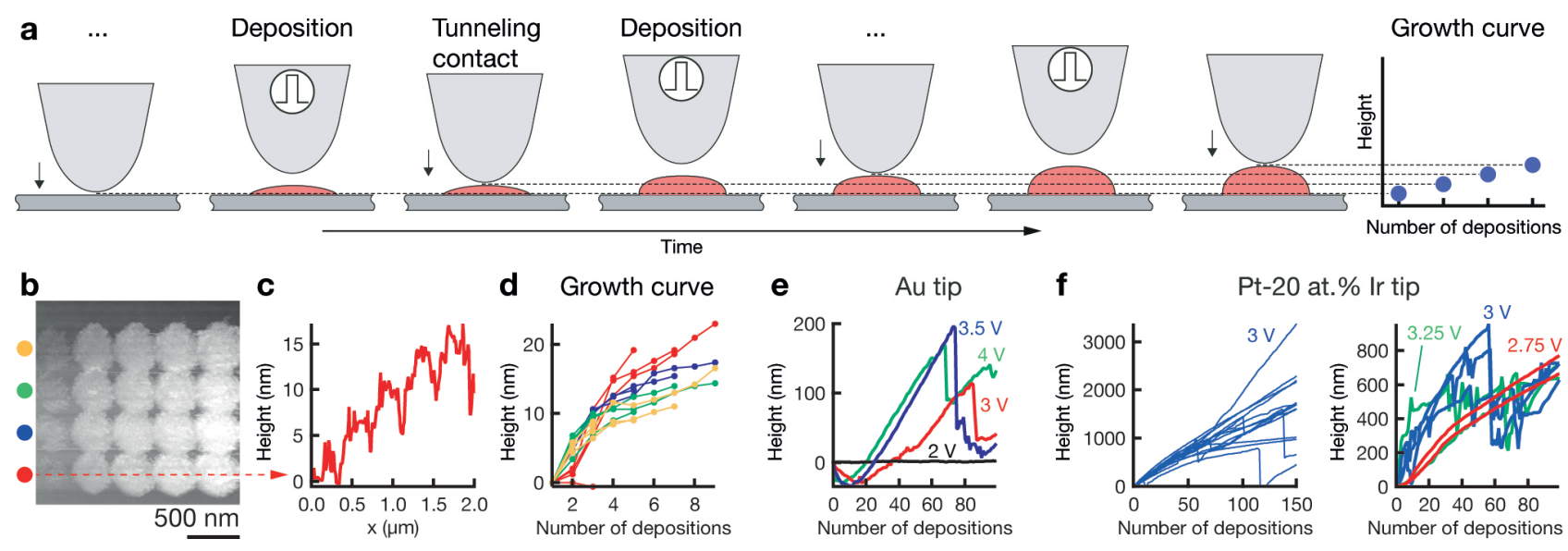

Figure 3: Towards 3D: out-of-plane growth. a, Repetition of single deposition events at the same location enables sequential "stacking" of deposits. The growth of the deposit can be monitored by recording the position of contact between tip and deposit during repositioning of the tip after each deposition event. b, STM scan of a grid of dots deposited with an increasing number of consecutive deposition events per point (left to right, from one to nine in steps of two). c, Corresponding crosssection line profile of the bottom row. d, Growth curves for each of the dots in (b). Colors correspond to the four rows in (b). e, Growth curves for a larger number of deposition events as a function of pulse amplitude obtained with a Au tip. Note the absence of growth for a pulse amplitude of $2 \mathrm{~V}$ (black, no deposition) and the initial, negative values at higher pulse voltages indicating etching of the tip. $\mathbf{f}$, Growth curves recorded with a Pt-20at.\%Ir tip in an aqueous electrolyte. Stable growth up to a height of 2-3 $\mu \mathrm{m}$ can be achieved (left), but growth curves are not free of instabilities. Curves recorded for pulse amplitudes of 2.75, 3 and $3.25 \mathrm{~V}$ (right) show that low amplitudes enable continuous growth, while larger pulse voltages cause growth instabilities. Deposition parameters. Electrolyte: $1 \mathrm{M}$ $\mathrm{CoSO}_{4}$ in DMSO (b-e), $1 \mathrm{M}$ aq. $\mathrm{CoSO}_{4}$ (f); pulse length: $100 \mathrm{~ns}$ (b-e), $20 \mathrm{~ns}$ (f); pulse amplitude: $4 \mathrm{~V}$ (b); duty cycle: 0.01; pulses per deposition event: 3000 (b-e), 10000-30000(f); dwell distance: $10 \mathrm{~nm}$ (b-e), $50 \mathrm{~nm}$ (f); dwell time: $0.2 \mathrm{~s}$ (b-e), $0.5 \mathrm{~s}$ (f); tip: Au (b-e), Pt-20at.\% Ir (f). 
of the growth curves to negative height values. Subsequently, the values typically increase, but are interrupted by sudden, pronounced drops. The initial drop is well explained by etching of Au tips, as described in Supplementary Section 2.1 (note the steady surface detection at $2 \mathrm{~V}$, indicating absence of tip crashes). After an initial incubation time, the out-of-plane growth rate of the deposit apparently outnumbers the etching rate, resulting in net positive values of the growth curve. The sudden drops in the growth curves are more difficult to assess. Recovery of growth after each drop speaks against a sudden and severe degradation of the tip, as this would likely cause termination of the experiment. We hypothesize that a shortcut between growing deposit and tip results in an instability of potential control and instantaneous dissolution of large parts of the grown deposit. Shortcuts were sometimes observed during application of pulses, presumably due to a contact of the growing deposit with the stationary tip. While we have not recorded the pulse current for these experiments, a shortcut caused by a deposit growing too fast could in principle be avoided by a continuous positioning feedback (during pulse off-time), and undesired swings in potential caused by a shortcut can be prevented by pausing potentiostat feedback during pulsing, as regularly employed in electrochemical machining.

Growth curves recorded for a Pt-20at.\% Ir tip in aq. $\mathrm{CoSO}_{4}$ suggest an increased process stability compared to Au tips (Figure 3 ) - single deposits reach heights of $1-3 \mu \mathrm{m}$ after $150 \times 30000$ pulses $(3 \mathrm{~V})$, and the number of growth curves that contain drops is decreased compared to experiments with Au tips. Nonetheless, abrupt collapses still occur with Pt-20at.\%Ir tips. These instabilities seem to be a function of pulse amplitude. A pulse amplitude of $2.75 \mathrm{~V}$ results in continuous growth, while growth curves for higher voltages feature the previously observed drops. A comparison of pre- and post-deposition micrographs of the utilized tip's apex still indicates loss of material, as expected from the growth curves (Supplementary Figure S9]. It is still to be shown that Pt or Pt-20at.\%Ir tips do not degrade with appropriate process parameters.

\section{What determines the feature size?}

The resolution of electrochemical machining is generally well described by a model based on the one-dimensional charging time constant of the double layer ${ }^{23 / 25 / 27}$. In our work, the qualitative influence of the machining parameters pulse length, electrolyte resistance, and dwell distance show the expected trends and corroborate importance of the general concept of local charging of the double layer (Figure 4). Shortening pulse widths from 100 to $20 \mathrm{~ns}$ results in a decrease of the diameter of single Co deposits from $\approx 500 \mathrm{~nm}$ to $\approx 200 \mathrm{~nm}$ in an aqueous electrolyte (Figure 4 $\mathrm{a}$ ), and from $\approx 150 \mathrm{~nm}$ to $\approx 50 \mathrm{~nm}$ in the DMSO electrolyte (Figure 4b). Further, an increase in electrolyte resistance decreases the width of deposits. A comparison of Co dots deposited from $2 \mathrm{M}$ aq. $\mathrm{CoSO}_{4}$ $(\rho \approx 20 \Omega \mathrm{cm})^{\frac{28}{28}}$ and $1 \mathrm{M} \mathrm{CoSO}_{4}$ in DMSO $(\rho \approx 140 \Omega \mathrm{cm})^{\sqrt{24}}$ using 20 ns pulses reveals a decrease in diameter from $\approx 200 \mathrm{~nm}$ to $\approx 75 \mathrm{~nm}$ (Figure 4c, d). Finally, an increased distance between tip and substrate results in a notable decrease of the lateral size of the deposits and a pronounced decrease of their volume (Figure 4e, f). Coarsely optimizing all the mentioned factors resulted in the smallest features obtained up to date: the elliptical deposit in Figure $4 \mathrm{p}$ ( $8 \mathrm{~ns}$ ), with a FWHM of 25 and $58 \mathrm{~nm}$ for the short and long axis, respectively. Note that no tip deconvolution was performed.

A quantitative comparison of the observed resolution of patterning shows that an approximation 


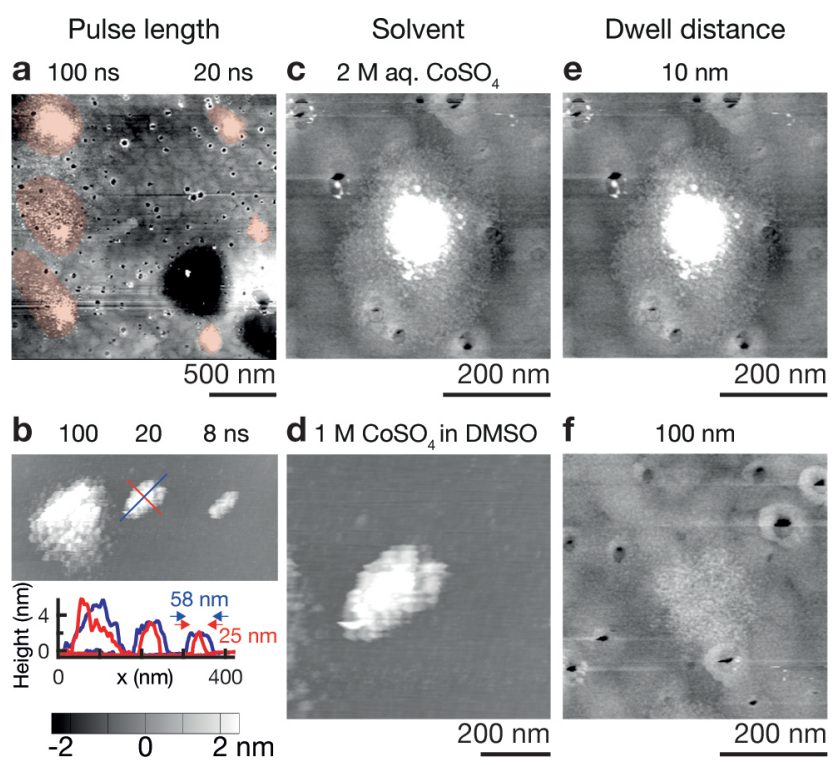

Figure 4: Parameters influencing the 2D feature size. The post-deposition STM scans compare the influence of a single parameter on the lateral extent of the deposit grown with otherwise unchanged parameters. a, b, The longer the polarizing pulses, the larger the resulting deposits from aqueous electrolytes (a) as well as DMSO-based electrolytes (b). The areas of the deposits are shaded in red as a guide for the eye in (a). The line profiles in (b) indicate the width along the long (blue) and the short (red) axes of the ellipsoid deposits. $\mathbf{c}, \mathbf{d}$, An increase in electrolyte resistance, i.e. when using DMSO (d) instead of water (c) as a solvent, increases the confinement for a given pulse length (20 ns, number of pulses: 500 (c), 50000 (d)). e, f, The lateral size of the deposits decreases with dwell distance (e, $10 \mathrm{~nm}, \mathrm{f}, 100 \mathrm{~nm})$. The greyscale height scale applies to all STM scans. Deposition parameters. Electrolyte: $1 \mathrm{M} \mathrm{CoSO}_{4}$ in DMSO (b, d), $2 \mathrm{M}$ aq. $\mathrm{CoSO}_{4}$ (a, c, e, f); pulse length: 20 ns (c-f); pulse amplitude: $5 \mathrm{~V}$ (a, c, e, f), $6 \mathrm{~V}$ (b, d); duty cycle: 0.01; pulses per point: 100 (a, $100 \mathrm{~ns}$ ), 500 (a, $20 \mathrm{ns,}$ c, e, f), 5000 (b, $100 \mathrm{ns)} 50000$ (b, $20 \mathrm{~ns}$, d) 800000 (b, $8 \mathrm{~ns}$ ) ; dwell distance: $10 \mathrm{~nm}$ (a, b, c, d, e), $100 \mathrm{~nm}(\mathrm{f})$. 
based on the 1D time constant of double layer charging significantly overestimates the deposit size, both in aqueous and DMSO electrolytes (by less than an order of magnitude though). For a specific electrolyte resistance of $\rho \approx 20 \Omega \mathrm{cm}\left(2 \mathrm{M}\right.$ aq. $\left.\operatorname{CoSO}_{4}{ }^{[28}\right)$ and $\rho \approx 140 \Omega \mathrm{cm}(1 \mathrm{M} \mathrm{LiCl}$ in DMSO 24 , for a rough estimate of $1 \mathrm{M} \mathrm{CoSO}_{4}$ in DMSO), we estimate significant charging during a $20 \mathrm{~ns}$ pulse $(\tau=20 \mathrm{~ns})$ to a distance $d=\tau /\left(\rho \times c_{\mathrm{DL}}\right)$ of $\approx 1 \mu \mathrm{m}$ (water) and $\approx 150 \mathrm{~nm}$ (DMSO) (double layer capacitance of $c_{\mathrm{DL}}=10 \mu \mathrm{F} \mathrm{cm}^{-2}$ ). In contrast, the approximate diameter of single Co deposits obtained with $20 \mathrm{~ns}$ pulses is $200 \mathrm{~nm}$ in water and $50 \mathrm{~nm}$ in DMSO (Figure 4k, d) (Typical tip radius of $50 \mathrm{~nm}$, dwell distance of $10 \mathrm{~nm}$ ). Note that unsatisfactory stability of the Au STM probes and thus a changing shape and size of the tip renders quantitative analysis of the present data questionable. Nonetheless we can state that the observed feature size is smaller than that predicted by the previous model because a blunting of the tip would lead to larger rather than smaller deposits. In further support of these observations, we report similar findings using more stable Pt tips in another publication 29 .

Thus, additional mechanisms of confinement must be at play. We hypothesize that the asymmetric electrode geometry and a significant nucleation overpotential caused by the short pulse time further limit the deposition in our favor. First, the 1D model of double layer charging assumes a parallel plate geometry and thus a ratio of area and capacitance of the two electrodes equal one - a given charge charges equal areas to equal potentials on both electrodes. This geometry closely represents the tool-substrate gap in micromachining and can be used to coarsely approximate the situation in the tip-substrate gap in our case. However, outside the tip-substrate gap, the ratio of tip area versus substrate area quickly becomes unfavourable for substrate charging — with increasing distance from the center, the rotational symmetry results in asymmetric charging of electrode areas connected by field lines, with the substrate being charged to a lower potential than the tip due to the ratio of surface area $A_{\text {substrate }} / A_{\text {tip }} \ll 1$. In other words, the local overpotential on the substrate is actually smaller than that predicted by the simple $1 \mathrm{D}$ charging model which infers the local overpotential from the mere distance to the tip and ignores the ratio of surface area. Because of the expected exponential relationship of growth rate to local overpotential, local growth rate will rapidly decrease for a distance larger than one tip radius $(\approx 50 \mathrm{~nm})$ from the center of a deposit. Second, in contrast to etching, deposition requires the formation of nucleii of critical size during polarization time, necessitating the accumulation of a critical Co adatom concentration on the substrate during the pulse. Thus, we might very well expect that there is a minimum local deposition current to guarantee formation of stable nucleii during nanosecond pulse times, and that this current - an exponential function of the potential - is only met where highest overpotentials are reached in the center of the polarized zone. Thus, the nucleation barrier may effectively reduce the area that experiences charging sufficient for deposition and thus increase the resolution of the deposition method.

Where is the limit of resolution then? We assume that a certain pulse width $t$ charges the substrate to a potential $U_{\mathrm{C}}$ sufficient for deposition within a distance $d$ from the tip surface. With a spherical tip of radius $r$ placed in front of a planar substrate, the width of the charged area then is described by $W=2 \sqrt{(r+d)^{2}-(r+D)^{2}}$, with $r$ the tip radius and $D$ the dwell distance for $W$ on the order of $2 r$ (Figure 5a), assuming a strictly radial current distribution in electrolyte. Note that an important parameter, the pulse amplitude - directly affecting the local overpotential and thus, in our model, the 
charging distance $d$ within which deposition occurs - is ignored in this simple model. Figure 5b, c plots $W$ as a function of $d, D$ and tip radius $r$. $W$ can be minimized by decreasing the tip radius, the charging distance $d$ or increasing the dwell distance $D$. Note that for any tip radius $r, W$ becomes zero for $d=D$. This limit is unphysical, since for $d D$ the triangle in Figure 5 a becomes progressively acute and the lateral variation of the surface potential will ultimately vanish. However, from Figure $5 \mathrm{~b}$ it can be seen that for all tip radii the width of the structures will decrease with decreasing charging distance, that is, for shorter pulses. Yet, a significant reduction of pulse width will become technically and experimentally challenging to implement. Thus, an optimization of the dwell distance is an attractive alternative, because it can be experimentally realized with relative ease. Albeit our model will break down when the dwell distance reaches the charging distance, from Figure 5 it can be seen that the width of the deposits strongly decreases with increasing dwell distance. We can coarsely predict a realistically achievable minimal resolution based on an experimentally verified charging distance $d=15 \mathrm{~nm}$ (Assuming a simplified circular geometry for the smallest deposit achieved in Figure $4 \mathrm{~b}$, a dwell distance of $10 \mathrm{~nm}$ and an approximate tip radius of $50 \mathrm{~nm}$ ). Using $d=15 \mathrm{~nm}$, let us place the tip at a realistic dwell distance of $D=12 \mathrm{~nm}<d$. With a present tip radius of $50 \mathrm{~nm}$, we could expect a width of $40 \mathrm{~nm}$ for the deposits. With a tip radius of $10 \mathrm{~nm}$, obtainable with optimized etching procedures or FIB milling, the minimal feature size would drop to approximately $25 \mathrm{~nm}$. Based on this prediction, a minimal feature size on the order of $10 \mathrm{~nm}$ - which would approach that of electron-beam-based techniques - seems realistic with further optimization of tip radius, dwell distance and pulse width.
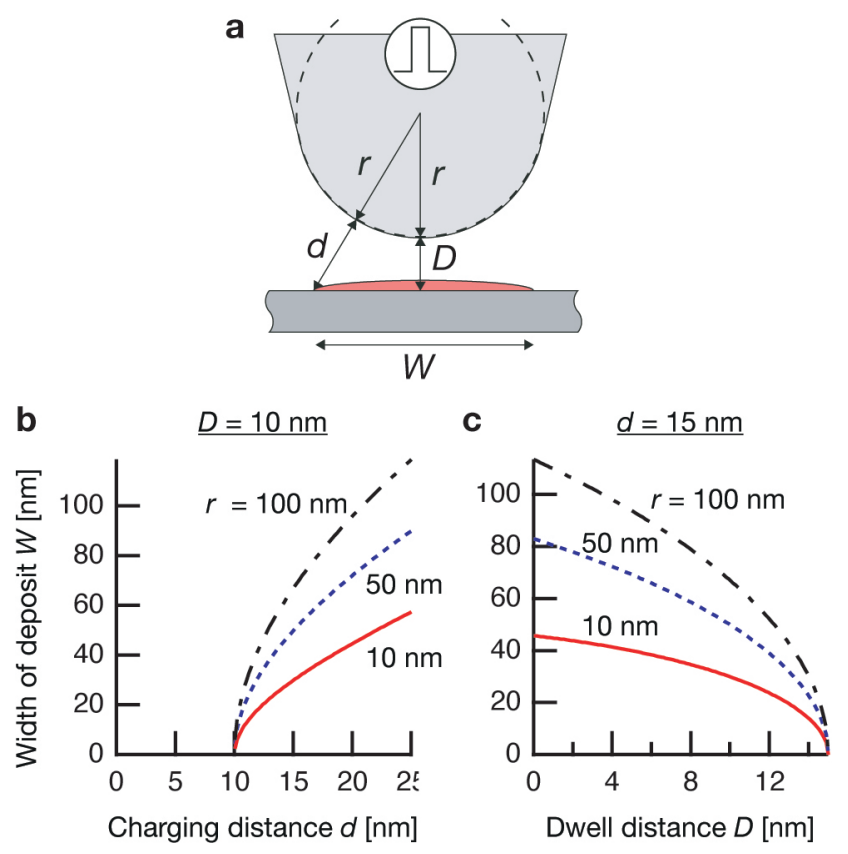

Figure 5: Predicted width of a deposit. a, Sketch of tip-substrate geometry. b, c, Predicted width of deposits $W=2 \sqrt{(r+d)^{2}-(r+D)^{2}}$ (with tip radius $r$, dwell distance $D$, and distance from tip $d$, up to which the substrate is charged sufficiently for deposition during a pulse) as a function of $\mathbf{b}$, charging distance $d$ (with $D=10 \mathrm{~nm}$ ), and $\mathbf{c}$, dwell distance $D$ (with $d=15 \mathrm{~nm}$, which is experimentally observed in Figure $4 \mathrm{p}$ ). 


\section{General advantages and limitations of the concept}

Confining substrate polarization during nanosecond voltage pulses is a robust approach for the strong localization of electrochemical reactions at the nanoscale (demonstrated herein for additive patterning, and previously for subtractive machining $25(27)$. In combination with easily available nanometer sized probes, a minimal feature size of $10-50 \mathrm{~nm}$ is probably within reach. As the method is based on electrochemical reduction and utilizes precursors of high purity, one can expect a materials performance similar to that of other electrochemical methods ${ }^{9}$. Thus, the concept could be attractive for niche applications that demand metals of high purity at smallest resolutions. A further advantage is the inherent growth feedback provided by all SPM based printing techniques - as shown in other work ${ }^{14}$, such a feedback can enable controlled deposition of most complex geometries.

Limitations of the concept are similar to those of other electrochemical techniques. First, a growth rate on the order of $10 \mathrm{~nm} \mathrm{~s}^{-1}$, i.e., $0.1 \mathrm{voxel} / \mathrm{s}$ for a typical feature size of $100 \mathrm{~nm}$ is comparable to deposition speeds of average FEBID and some electrochemical techniques ${ }^{5}$, but low compared to up to $10 \mathrm{voxel} / \mathrm{s}$ for electrohydrodynamic redox printing ${ }^{4}$ (currently limited to a minimal feature size of about $100 \mathrm{~nm}$ ) and tens and hundreds of voxel/s for non-electrochemical techniques ${ }^{5}$ (many of them with a minimal feature size of $\approx 1 \mu \mathrm{m}$.

Note that the growth rate is currently slowed down by a deposition procedure that is not optimized for speed, with significant delays added by the slow movement of the tip. For example, the summed pulse period time (pulse on and off time) for the $200 \mathrm{~nm}$ grown in 75 depositions in Figure 3 was only $2.25 \mathrm{~s}$, compared to $40 \mathrm{~s}$ needed to actually execute 75 depositions. Similarly, total pulse period time in Figure $3 \mathrm{f}$ was $3 \mathrm{~s}$ versus a total process time of $150 \mathrm{~s}$. Consequently, an increase in speed by a factor of ten is realistic for optimized deposition procedures that limit the movement of the tip and thus interruptions in the deposition process. For example, one could imagine a steady retraction of the tip in combination with continuous pulsing. Further, an optimization of the low duty cycle required for deposition (0.01-0.1) and increased mass transport to re-supply the consumed ions in the tip-substrate gap could additionally increase deposition rate. For example, one can envision a combination of the method with concentration-confined electrodeposition $\frac{\sqrt{14}}{}$ which provides high rates of ion supply by pressure-dispensing a concentrated salt solution from a hollow AFM tip. A second limitation is the need for an electrically conductive substrate (although one can imagine wiring in-between electrodes, as shown for example with electrochemical electrohydrodynamic redox printing ${ }^{4}$ ). Finally, in contrast to other electrochemical methods, the application of the presented concept is simplified for an electrochemical system that is characterized by partly irreversible metal deposition that offers reduction and oxidation potentials that are separated by at least a few tens of $\mathrm{mV}$ (keeping the surface potential within this potential interval avoids delocalized deposition but guarantees the stability of the localized deposits). We demonstrated the application for Co deposition, but deposition of other metals, for example Ni, should be similarly feasible. Furthermore, the constraint towards irreversible electrochemical systems may be relaxed by careful control of the rest potentials of tip and surface, as demonstrated for local $\mathrm{Cu}$ deposition from acidic $\mathrm{CuSO}_{4}$ solutions ${ }^{23}$. 


\section{Conclusion}

We have confirmed that confinement of electrodeposition by application of nanosecond voltage pulses to a local probe such as an STM tip can be used for 2D surface patterning with smallest feature size of $\approx 50 \mathrm{~nm}$ as well as out-of-plane growth of Co structures. As in electrochemical micromachining, the limited spatial extent of double-layer charging is the main mechanism to localize the electrochemical reaction, and pulse length and tip-substrate distance are the main parameters that control resolution. Yet, we assume that additional mechanisms based on the asymmetric tip-substrate geometry and a high nucleation barrier during short pulses further favor localization of deposition below the probe. We demonstrated a feature size of about $50 \mathrm{~nm}$ for Co deposition from a DMSO-based electrolyte with a tip radius of about $50 \mathrm{~nm}$. With smaller tips, an optimized dwell distance during deposition and a moderate decrease of pulse width, a feature size on the order of $10 \mathrm{~nm}$ may be within reach, approaching values obtained with FEBID. Importantly, we identified issues with the stability of Au STM tips, and could demonstrate an enhanced inertness of Pt or Pt-20at.\%Ir probes - a finding that will improve reproducibility in future experiments. The study highlights that the stability of the tip in a large process window is of utmost importance for the explored concept. Without an inert behavior of the probe, its size and shape and thus the size and shape of the deposits will not be constant, rendering reliable patterning and quantitative analysis of the deposition mechanism impossible.

In comparison with other electrochemical small-scale AM methods, the concept offers potential for smallest feature size, but currently a growth rate on the order of $0.1 \mathrm{voxel} / \mathrm{s}$ only. Thus, potential application of the concept might be restricted to niche applications that demand highest resolution and high metal content. In applications that demand for direct, economic nanoscale patterning of pure metals on conductive surfaces, for example for photonic and plasmonic surfaces and 3D structures, the technique might present a future alternative to FEBID or lithographical patterning.

\section{Methods section}

\section{Electrochemical STM and pulse setup}

A schematic of the custom-built setup for additive deposition with nanosecond voltage pulses is shown in Supplementary Figure S1. The setup is composed of three subsystems: an STM (Cypher-AFM in STM-mode, Asylum Research, USA) combined with a custom-built electrochemical cell, a bipotentiostat (PGUmicro, IPS, Germany) and a pulse pattern generator (2x81111A, Agilent, USA). The purpose of the STM is twofold: first, it controls the movement of the substrate relative to the tip in three dimensions during deposition. Second, it provides the tunneling current as a position feedback signal. This feedback allows the positioning of the tip before and during deposition with sub-nanometer precision. The bi-potentiostat controls the average potentials of the tip and the substrate - both immersed in an electrolyte solution - versus the reference electrode and thereby governs global, non-localized electrochemical reactions. Additionally, it monitors the corresponding faradaic currents. In contrast, the pulse generator controls the potential of the tip and the substrate at MHzrates, inducing localized electrochemical reactions under the tip. The pulse amplitude and the pulse 
current are monitored with an oscilloscope (6404D, PicoScope, England). It is important to note that the measurement grounds of the three subsystems are held at the same level to avoid leakage currents between the individual instruments. This is achieved on a hardware level by shorting the BNC ground connections of the instruments and by isolating the bi-potentiostat from mains earth using an isolation transformer.

The setup operates in two distinct modes that cannot run simultaneously, namely a feedback mode and a pulse mode. In feedback mode, the STM tip is connected to the input of the STM current amplifier. This enables the monitoring of the tunneling current and hence precise positioning of the tip. The tunneling voltage is equal to the potential of the tip (Working electrode 1, WE1) and the surface potential of the substrate (WE2). In pulse mode, the tip is routed to the output of the pulse generator, so that voltage pulses can be applied to the tip. A mechanical relay switch (Supplementary Figure S1 a, (2)) switches the electrical connection of the tip between the STM current input and the pulse generator as required. In either position of the switch, the rest potential of the tip is virtual ground.

The current circuits for low- (blue) and high-frequency currents (red) are separated by appropriate high- and low-pass filters (Supplementary Figure $\mathrm{S}_{1} \mathrm{a},(4,5)$ ). The low-frequency currents, representing global faradaic reactions, flow between the counter electrode (CE) and the tip (for faradaic reactions at the tip) or between the CE and the substrate connected to the WE2 input of the potentiostat (faradaic reactions at the substrate). The high-frequency current flows from (or into) the output of the pulse generator through the tip into the substrate and subsequently across a capacitance (high-pass filter, (4)) to ground. In order to minimize the interference of these two circuits, low-pass filters (5) are connected to RE, CE and WE2 in series (see Figure SS2 for a schematic of the electronic circuits).

The deposition procedure is computer controlled through a customized software developed inhouse and programmed in the Igor Pro software environment (Wavemetrics). It facilitates point-wise or continuous deposition, implements a correction for substrate tilt, and enables recording of out-ofplane growth curves.

\section{Chemicals and Materials}

The electrochemical cell was designed in-house and made from polychlorotrifluoroethylene (PCTFE, Cellpack, Switzerland). O-rings were purchased from Angst+Pfister (NORMATEC FKM 70, Switzerland). The counter- and quasi-reference-electrode were plain Au wires ( $0.1 \mathrm{~mm}$ diameter, Möller AG). All constituents of the cell and the electrodes were immersed in fresh piranha solution (3:1 volumetric mixture of $\mathrm{H}_{2} \mathrm{SO}_{4}$ (conc.) : $\mathrm{H}_{2} \mathrm{O}_{2}(30 \%)$ ) and subsequently rinsed with MilliQ water and stored in MilliQ water until used. Substrates were either Au single crystals ((111)-out-of-plane $<1 \%$, $99.999 \%$ purity, Mateck, Germany) or $100 \mathrm{~nm}$ thick Au films evaporated onto Si-wafers (including a $\mathrm{Cr}$ adhesion layer, $10 \mathrm{~nm}$ ). Single crystals were flame-annealed in a propane-butane flame for $10 \mathrm{~min}$ preceding every experiment. After each experiment, the single crystals were cycled in $1 \mathrm{M}$ aqueous $\mathrm{H}_{2} \mathrm{SO}_{4}$ to remove metallic residues.

Au STM tips were electrochemically etched from Au wires $(0.25 \mathrm{~mm}$ diameter, $99.999 \%$, Alfa Aesar, Germany) in a 1:1 volumetric solution of $\mathrm{HCl}$ (fuming) and ethanol. For etching, we used a 
potentiostat (PTSTAT12, Metrohm Autolab, Netherlands) to apply a positive DC potential of $2.4 \mathrm{~V}$ between a Pt counter-electrode and the Au wire. No current monitor or feedback mechanism to terminate etching was used. After etching, the tips were rinsed with MilliQ water and blown-dried. Typical radii of Au tips were $50 \mathrm{~nm}$. Pt tips were etched from Pt wires (0.25 mmdiameter, 99.997\%, Alfa Aesar, Germany) in aq. $\mathrm{NaOH}$ solutions (DC or AC voltage profiles with various amplitudes $(2-10$ V)). Pt-20at.\%Ir tips were purchased from NaugaNeedles (NN-USPtIr-W250, nominal tip radius $25-50 \mathrm{~nm}$ ). All STM tips were coated with a thermoplastic wax (Apiezon, England) to minimize the active surface area of the tips to approximately $50 \mu \mathrm{m}^{2}$.

Electrolytes were prepared in glass vials previously cleaned in fresh piranha solution and thoroughly washed with MilliQ water. Typical concentrations were 1 and $2 \mathrm{M}$ of $\mathrm{CoSO}_{4}$ (Heptahydrate, 99.999 \%, Alfa Aesar, Germany). The solvent was either MilliQ water or dimethyl sulfoxide (DMSO, analytical reagent grade, Fisher Scientific UK). The electrolyte was handled with sterile plastic Eppendorf pipette tips. All experiments were conducted under atmospheric conditions, without any purging of the electrolytes.

\section{Analysis}

The dimensions of the deposits were typically assessed in situ via STM imaging (typical parameters were: tunneling current: $\approx 1 \mathrm{nA}$, tunneling voltage: $\approx 200 \mathrm{mV}$, scan rate: $1-2 \mathrm{~Hz}$ ). Section line profiles were always averaged over a width of 10 scan lines. SEM analysis was performed with a Magellan 400 SEM (FEI, USA).

\section{References}

[1] Sattelkow, J. et al. Three-Dimensional Nanothermistors for Thermal Probing. ACS Appl. Mater. Interfaces 11, 22655-22667 (2019).

[2] Arnold, G. et al. Tunable 3D Nanoresonators for Gas-Sensing Applications. Adv. Funct. Mater. 28, 1707387 (2018).

[3] Kuhness, D. et al. High-Fidelity 3D Nanoprinting of Plasmonic Gold Nanoantennas. ACS Appl. Mater. Interfaces 13, 1178-1191 (2021).

[4] Reiser, A. et al. Multi-metal electrohydrodynamic redox 3D printing at the submicron scale. Nat. Commun. 10, 1853 (2019).

[5] Hirt, L., Reiser, A., Spolenak, R. \& Zambelli, T. Additive Manufacturing of Metal Structures at the Micrometer Scale. Adv. Mater. 201604211, 1604211 (2017).

[6] Botman, A., Mulders, J. J. L. \& Hagen, C. W. Creating pure nanostructures from electron-beaminduced deposition using purification techniques: a technology perspective. Nanotechnology $\mathbf{2 0}$, 372001 (2009). 
[7] Lukasczyk, T., Schirmer, M., Steinrück, H.-P. \& Marbach, H. Electron-Beam-Induced Deposition in Ultrahigh Vacuum: Lithographic Fabrication of Clean Iron Nanostructures. Small 4, 841-846 (2008).

[8] Utke, I. et al. Focused electron beam induced deposition of gold. J. Vac. Sci. Technol. B Microelectron. Nanom. Struct. 18, 3168 (2000).

[9] Reiser, A. et al. Metals by Micro-Scale Additive Manufacturing: Comparison of Microstructure and Mechanical Properties. Adv. Funct. Mater. 30, 1910491 (2020).

[10] Rohner, P. et al. 3D electrohydrodynamic printing and characterisation of highly conductive gold nanowalls. Nanoscale 12, 20158-20164 (2020).

[11] Schneider, J. et al. Electrohydrodynamic NanoDrip Printing of High Aspect Ratio Metal Grid Transparent Electrodes. Adv. Funct. Mater. 26, 833-840 (2016).

[12] Skylar-Scott, M. A., Gunasekaran, S. \& Lewis, J. A. Laser-assisted direct ink writing of planar and 3D metal architectures. Proc. Natl. Acad. Sci. 113, 6137-6142 (2016).

[13] Hengsteler, J. et al. Bringing Electrochemical Three-Dimensional Printing to the Nanoscale. Nano Lett. 21, 9093-9101 (2021).

[14] Hirt, L. et al. Template-Free 3D Microprinting of Metals Using a Force-Controlled Nanopipette for Layer-by-Layer Electrodeposition. Adv. Mater. 28, 2311-2315 (2016).

[15] Momotenko, D., Page, A., Adobes-Vidal, M. \& Unwin, P. R. Write-Read 3D Patterning with a Dual-Channel Nanopipette. ACS Nano 10, acsnano.6b04761 (2016).

[16] Schindler, W., Hofmann, D. \& Kirschner, J. Localized electrodeposition using a scanning tunneling microscope tip as a nanoelectrode. J. Electrochem. Soc. 148, C124-C130 (2001).

[17] Schuster, R., Kirchner, V., Xia, X. H., Bittner, a. M. \& Ertl, G. Nanoscale Electrochemistry. Phys. Rev. Lett. 80, 5599-5602 (1998).

[18] Maynor, B. W., Li, Y. \& Liu, J. Au “Ink” for AFM “Dip-Pen” Nanolithography. Langmuir 17, 2575-2578 (2001).

[19] Obermair, C., Kress, M., Wagner, A. \& Schimmel, T. Reversible mechano-electrochemical writing of metallic nanostructures with the tip of an atomic force microscope. Beilstein J. Nanotechnol. 3, 824-830 (2012).

[20] Jansson, A., Thornell, G. \& Johansson, S. High Resolution 3D Microstructures Made by Localized Electrodeposition of Nickel. J. Electrochem. Soc. 147, 1810 (2000).

[21] Li, W., Virtanen, J. A. \& Penner, R. M. Nanometer-scale electrochemical deposition of silver on graphite using a scanning tunneling microscope. Appl. Phys. Lett. 60, 1181-1183 (1992). 
[22] LaGraff, J. R. \& Gewirth, A. a. Nanometer-Scale Mechanism for the Constructive Modification of $\mathrm{Cu}$ Single Crystals and Alkanethiol Passivated Au(111) with an Atomic Force Microscope. J. Phys. Chem. 99, 10009-10018 (1995).

[23] Schuster, R., Kirchner, V., Allongue, P. \& Ertl, G. Electrochemical Micromachining. Science (80-. ). 289, 98-101 (2000).

[24] Ma, X., Bán, A. \& Schuster, R. Electrochemical Machining of Gold Microstructures in LiCl/Dimethyl Sulfoxide. ChemPhysChem 11, 616-621 (2010).

[25] Schuster, R. Electrochemical microstructuring with short voltage pulses. ChemPhysChem $\mathbf{8}$, 34-39 (2007).

[26] de Abril, O., Gündel, a., Maroun, F., Allongue, P. \& Schuster, R. Single-step electrochemical nanolithography of metal thin films by localized etching with an AFM tip. Nanotechnology 19, 325301 (2008).

[27] Kock, M., Kirchner, V. \& Schuster, R. Electrochemical micromachining with ultrashort voltage pulses-a versatile method with lithographical precision. Electrochim. Acta 48, 3213-3219 (2003).

[28] Bešter-Rogač, M. Electrical Conductivity of Concentrated Aqueous Solutions of Divalent Metal Sulfates. J. Chem. Eng. Data 53, 1355-1359 (2008).

[29] Aarts, M., Reiser, A., Spolenak, R. \& Alarcon-llado, E. Confined Pulsed Diffuse Layer Charging for Nanoscale Electrodeposition with an STM. ChemRxiv (2021).

\section{Author contributions}

AR wrote the control software and designed and built the STM-based deposition setup with support of R. Schuster. AR designed and performed experiments and their analysis. R. Spolenak advised AR's contribution to the work. AR drafted the manuscript, and all authors discussed the results and reviewed and commented on the text.

\section{Acknowledgments}

The authors thank the members of the machine shop of the department of materials, ETH Zürich, for their contribution to the setup. AR would like to thank Marting Grüßer for his support of preliminary experiments at Karlsruhe Institute of Technology, Mark Aarts (AMOLF, Netherlands), who has conducted the experiments shown in Figure 2d, e, as well as Steffen Geisel, Vladimir Vojtech and Johannes Gabl who evaluated various tip materials in their student projects. Electron-microscopy analysis was performed at ScopeM, the microscopy platform of ETH Zürich. The work was supported by ETH Zürich through grant No. ETH 47 14-2. 


\section{Supplementary Information}

\section{Experimental}

a

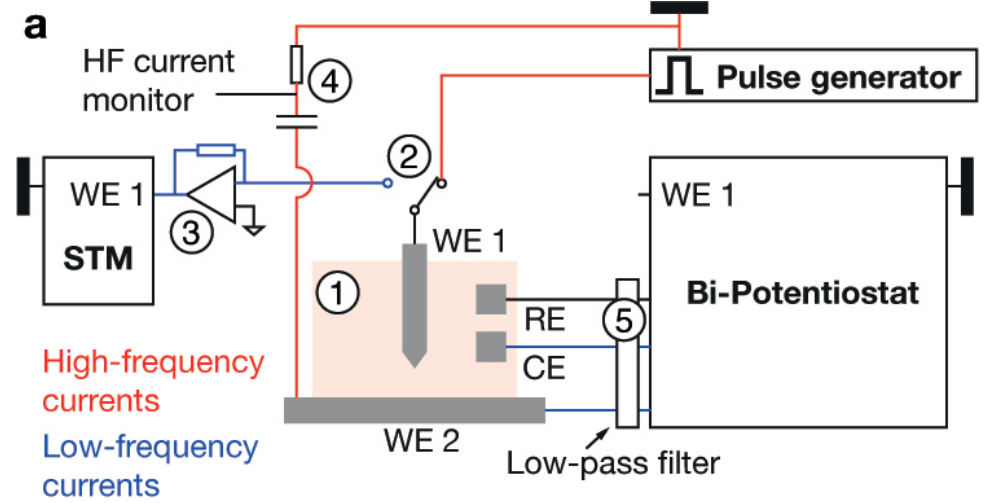

currents

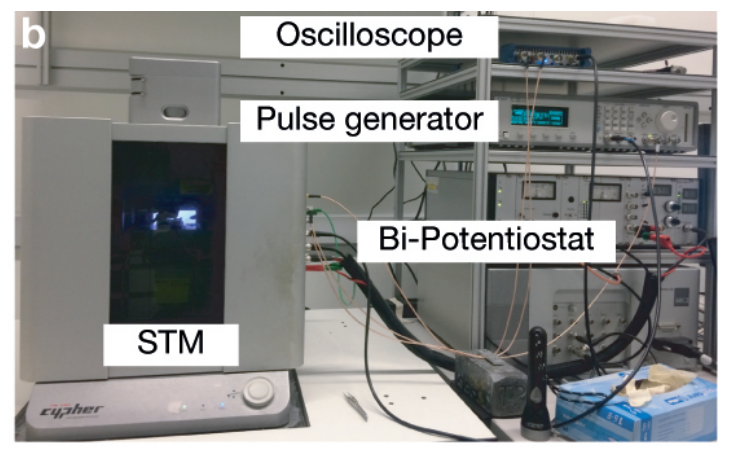

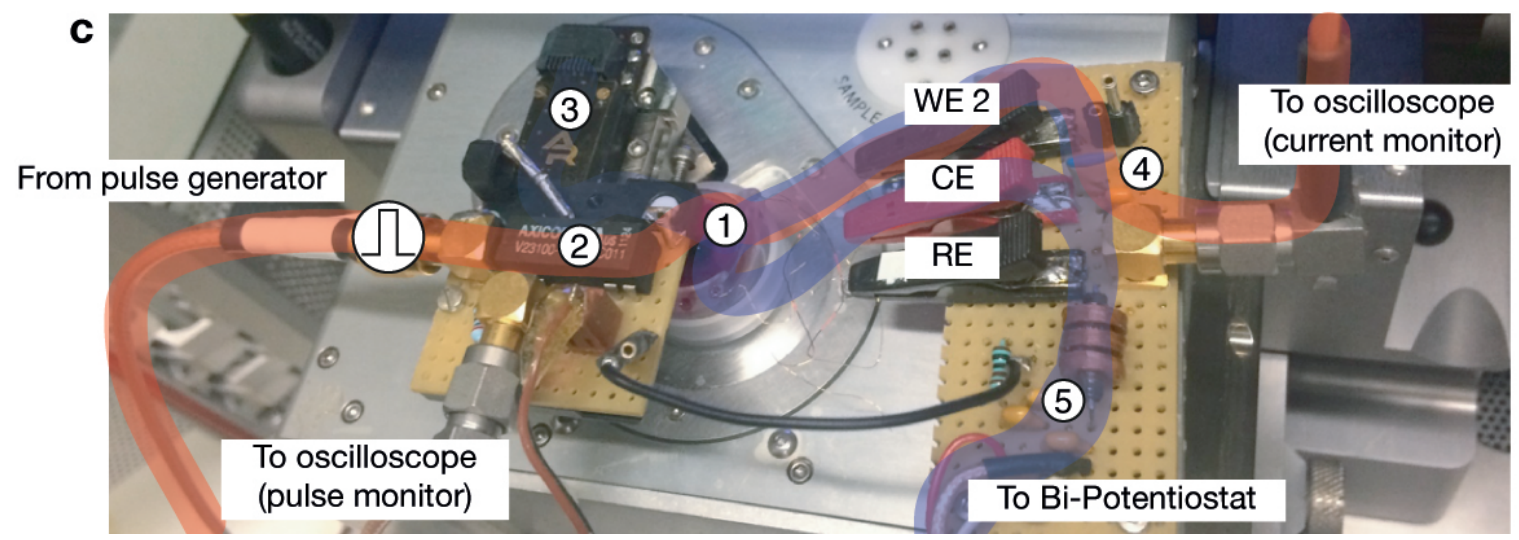

Figure S1: Setup for local deposition in an electrochemical STM. a, Schematic of the home-built setup and corresponding photographs of $\mathbf{b}$, the three sub-systems that were joined and $\mathbf{c}$, the electrochemical cell with corresponding STM and filter electronics. The main components are: STM tip (WE 1) and substrate (WE 2) are immersed in an electrolyte solution contained in an electrochemical cell (1). The surface potentials of WE 1 and 2 are controlled by a bi-potentiostat. The tip is either connected to the current input of the STM (3) or the output of the pulse generator and is switched between the two with a mechanical relay (2). A high-pass filter (4) and low-pass filters (5) separate the high-frequency (red) and the low-frequency (blue) current circuits. 


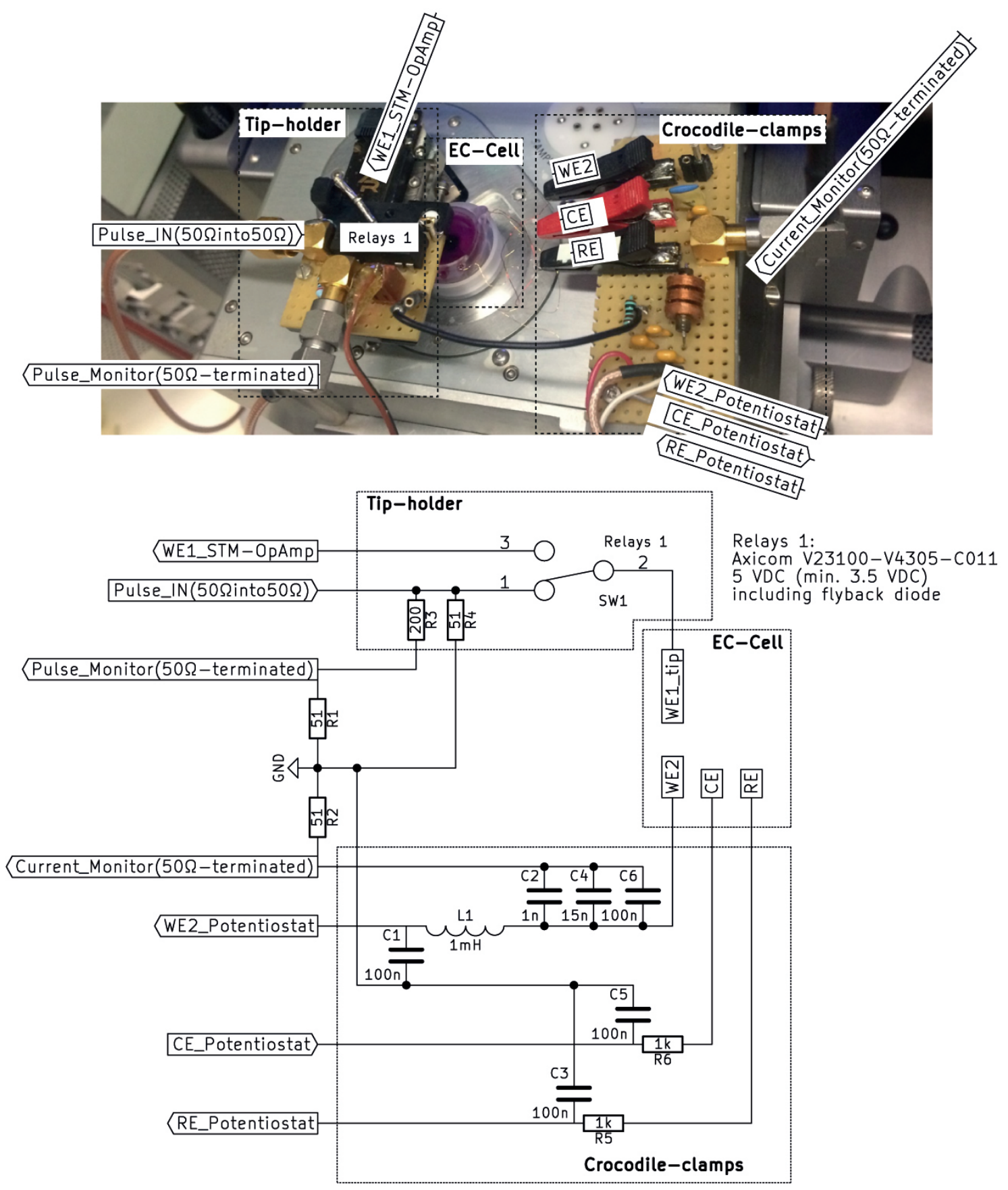

Figure S2: Circuit diagram of the electronic components that couple the nanosecond pulses into the electrochemical cell and separate the high- and low-frequency currents. Main in- and outputs as well as some key components are labelled in the photograph. 


\section{Results}

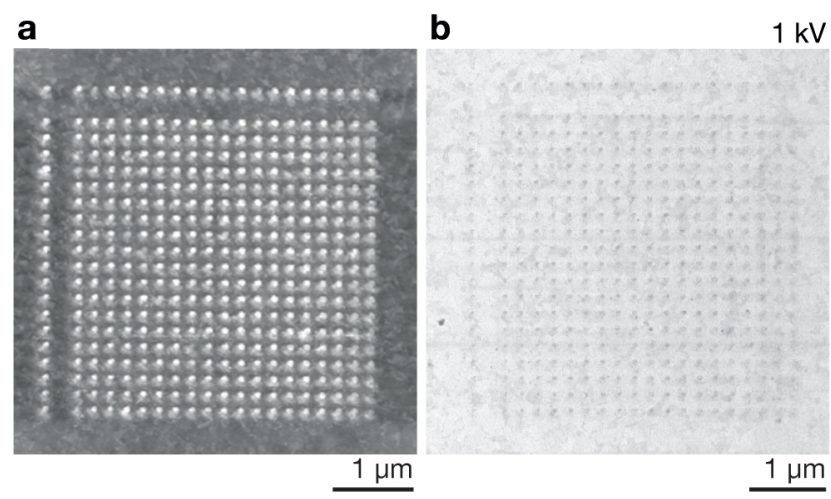

Figure S3: SEM micrograph of a 2D grid. a, STM scan and b, $1 \mathrm{kV}$ SEM micrograph of a grid (same as in Figure 2c). SEM EDX analysis of printed 2D patterns is not possible, as they are electrontransparent at acceleration voltages needed for analysis. Deposition parameters. Electrolyte: $1 \mathrm{M}$ $\mathrm{CoSO}_{4}$ in DMSO; pulse length: $100 \mathrm{~ns}$; pulse amplitude: $6 \mathrm{~V}$; duty cycle: 0.01 ; pulses per point: 3000; dwell distance: $10 \mathrm{~nm}$.

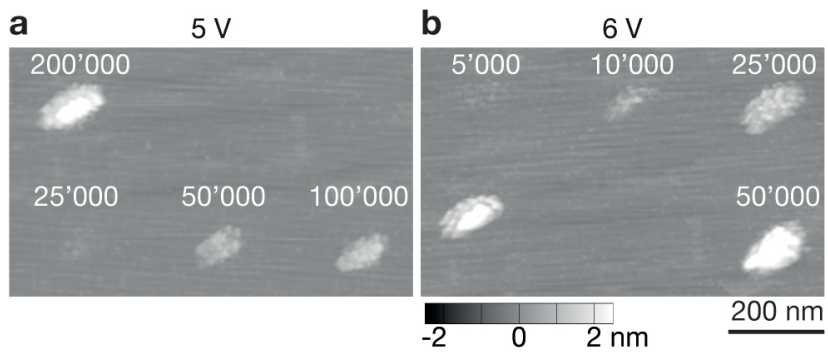

Figure S4: Influence of total pulse number per deposit. Deposits grown with increasing number of pulses (as stated in the images) but otherwise identical parameters, for a pulse amplitude of $\mathbf{a}, 5 \mathrm{~V}$ and b, $6 \mathrm{~V}$. At initial stages of deposition, with low numbers of pulses, the lateral diameter increases with the amount of pulses. For larger numbers of pulses, the lateral size of the deposits is approximately constant (compare 25000 and 50000 pulses in b), while its height increases. Note that the bottom-left deposit in (b) corresponds to the top-left deposit in (a). Deposition parameters. Electrolyte: $1 \mathrm{M}$ $\mathrm{CoSO}_{4}$ in DMSO; pulse length: $20 \mathrm{~ns}$; pulse amplitude: as stated; duty cycle: 0.01 ; pulses per point: as stated; dwell distance: $10 \mathrm{~nm}$.

\subsection{Insufficient stability of Au STM tips}

Negative values of the growth curves in Figure 3e indicate either the tip crashing into the substrate, a hole being milled into the substrate, or degradation of the tip. Post-deposition STM scans of the substrate exclude any subtractive machining of the substrate. Repeated tip crashes are also unlikely, as post-deposition SEM analysis of tips do not show any bending of the tip. However, a comparison of a Au STM tip before and after its use in multiple deposition experiments confirms removal of material from the tip apex (Figure $\mathbf{S 5} \mathrm{a}$ ), presumably due to anodic dissolution of $\mathrm{Au}$.

To verify the loss of tip material upon anodic pulsing, we designed a straightforward model experiment based on the assumption that any material that is dissolved from the tip would redeposit on the substrate. Pulse experiments are conducted in supporting electrolyte solutions containing no metal 

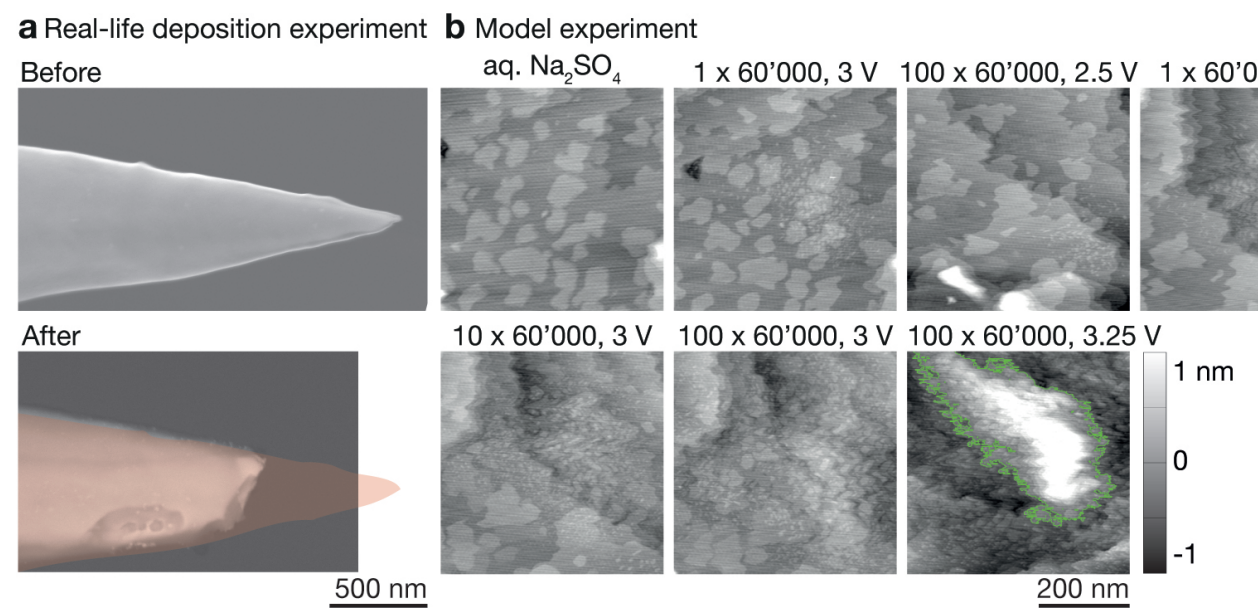

C

Before

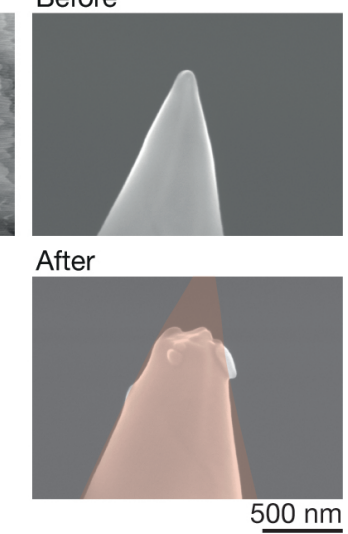

Figure S5: Anodic degradation of Au tips. a, SE SEM micrographs of a Au STM tip before (left) and after (right) its use as a local electrode for multiple deposition experiments. A length of $>500 \mathrm{~nm}$ of the original tip apex is missing after experiments. The red shading in the right micrograph indicates the dimensions of the original tip. b, c, A model experiment confirms substantial loss of tip material upon pulsing in aq. $0.25 \mathrm{M} \mathrm{Na}_{2} \mathrm{SO}_{4}$. As the electrolyte solution is bare of metal cations that could deposit on the substrate, the deposited material in must originate from the STM tip. b, STM scans of a $\mathrm{Au}$ surface before (first scan) and after pulsing the Au STM tip in $0.25 \mathrm{M}$ aq. $\mathrm{Na}_{2} \mathrm{SO}_{4}$ using the parameters stated in the panels (Pulse width: $20 \mathrm{~ns}$; duty cycle: 0.01 ; dwell distance: $50 \mathrm{~nm}$ ). The location of scans 3-7 is different from 1-2. The volume of the outlined deposit in the last scan is $6.3 \times 10^{4} \mathrm{~nm}^{3}$. c, SEM micrograph of the Au tip used in (a) before (left) and after (right) the deposition experiments. The estimated volume of the tip material missing after deposition is $\approx 2 \times 10^{7} \mathrm{~nm}^{3}$.

cations that could be deposited (typically $0.25 \mathrm{M}$ aq. $\mathrm{Na}_{2} \mathrm{SO}_{4}$ ). Consequently, all material grown on the substrate after a deposition event must originate from the tip. The used STM tips are imaged prior to and after deposition experiments. Indeed, these experiments confirm loss of tip material (Figure $[\mathrm{S} 5 \mathrm{~b}, \mathrm{c})$. Thus, the following assumption is made and applied to the rest of the result section. Any material deposited in $\mathrm{Co}^{2+}$-ion-free electrolyte solutions originates from the utilized tip and thus indicates the tip's degradation. However, this is no quantitative relation, as not all material dissolved from the tip transfers to the substrate. In Figure $\mathrm{S} 5 \mathrm{~b}, \mathrm{c}$, the estimated volume removed from the tip is $\approx 2 \times 10^{7} \mathrm{~nm}^{3}$, compared to only $\approx 6 \times 10^{4}-\max .1 \times 10^{5} \mathrm{~nm}^{3}$ for the largest deposit in (b).

\subsection{Sufficient stability of Pt and Pt-Ir STM tips}

The anodic instability of Au under typical deposition conditions necessitates an alternative tip material. A comparison of pulse experiments of $\mathrm{Au}$ and $\mathrm{Pt}$ tips in a $\mathrm{Co}^{2+}$-free supporting electrolyte suggest a higher stability of Pt (Figure S6. While first clear signs of Au tip degradation, i.e., deposited material below the tip, are detected after $1 \times 60000$ pulses, similar deposition of Pt is only observed after $100 \times 60000$ pulses (although there is minor deposition already after $10 \times 60000$ pulses).

The deposition of Pt tip material onto the substrate is minimized if the pulse amplitude is decreased to $2.5 \mathrm{~V}$ (Figure $\mathbf{S 7}$ a). This suggest a sufficient stability of the tip for Co deposition when compared to typical deposition parameters in $\mathrm{Co}^{2+}$-containing electrolyte solutions. Indeed, a depo- 

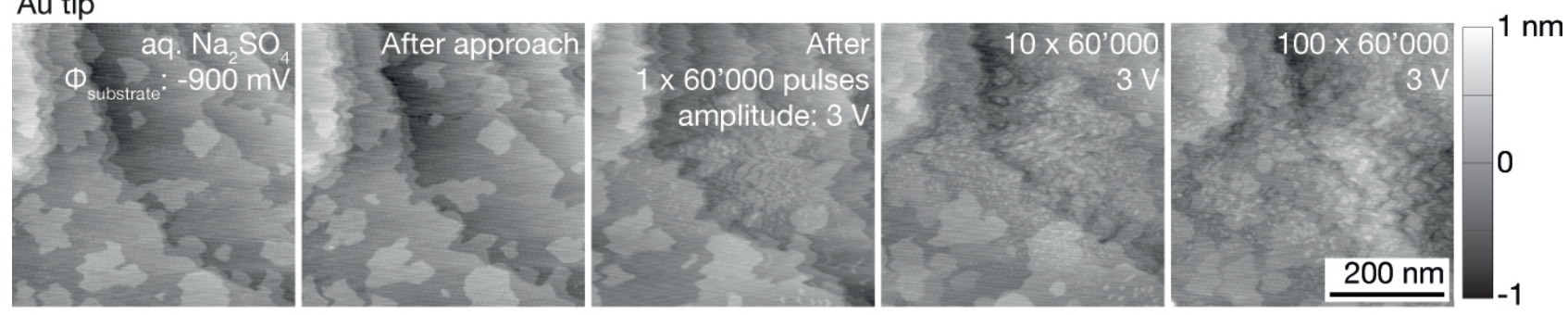

Pt tip
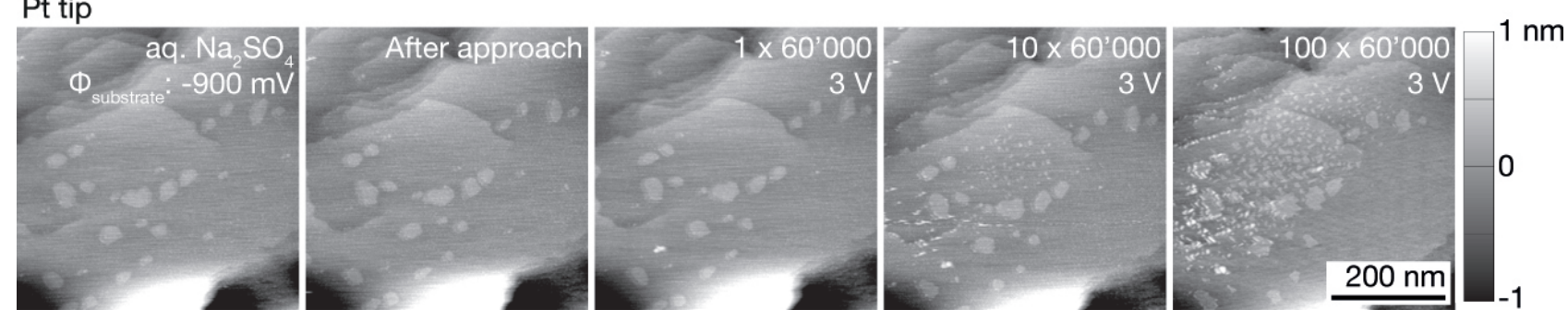

Figure S6: Anodic stability of Au versus Pt tips. STM scans of a Au surface before and after $1 \times$, $10 \times$ and $100 \times 60000$ voltage pulses applied to either a $\mathrm{Au}$ (top row) or a Pt tip (bottom row) in $0.25 \mathrm{M}$ aq. $\mathrm{Na}_{2} \mathrm{SO}_{4}$. For the Au tip, deposition is evident already after $1 \times 60000$ pulses, indicating dissolution of the tip. In contrast, a similar amount of deposition from the Pt tip is only observed for $100 \times 60000$ pulses, suggesting a higher resistance of Pt against anodic oxidation. The label OFF indicates a simple tip approach without pulses. Deposition parameters. Pulse width: $20 \mathrm{ns;}$ pulse amplitude: $3 \mathrm{~V}$; duty cycle: 0.1 ; dwell distance: $50 \mathrm{~nm}$; $\Phi_{\text {substrate }}:-900 \mathrm{mV}$; $\Phi_{\text {tip }}:-700 \mathrm{mV}$ vs. Au.

sition experiment in $0.25 \mathrm{M}$ aq. $\mathrm{CoSO}_{4}$ yielded pronounced deposition already at $1 \times 60000$ pulses (Figure $\left[\mathrm{S} 7 \mathrm{p}\right.$ ) - a total polarization time that does not deposit any tip material in $0.25 \mathrm{M}$ aq. $\mathrm{Na}_{2} \mathrm{SO}_{4}$ (a). Additionally, first signs of deposition are already evident after 5000 pulses. Lifting the surface potential to $\Phi_{\text {substrate }}>\Phi_{\mathrm{Co} \rightarrow \mathrm{Co}^{2+}}(-200 \mathrm{mV}$ vs. Au $)$, the deposit instantaneously dissolves and only some residues remain (Figure $\mathbf{S 8}$ ). Smaller dots grown with a lower total polarization time dissolve completely (Figure $\mathrm{S} 8 \mathrm{~b}$ ), indicating no co-deposition of Pt during pulsing.

The increased stability of Pt and Pt-20at.\%Ir tips suggests the potential for stable and controlled deposition. However, as preliminary results show, the tips' inertness is only guaranteed in a narrow process window. Growth curves recorded for a Pt-20at.\% Ir tip in aq. $\mathrm{CoSO}_{4}$ suggest an increased process stability compared to Au tips - single deposits reach heights of $1-3 \mu \mathrm{m}$ after $150 \times 30000$ pulses $(3 \mathrm{~V})$, and the number of growth curves that contain drops is decreased compared to experiments with Au tips (Figure S9]. Nonetheless, abrupt collapses still occur with Pt-20at.\%Ir tips. These instabilities seem to be a function of pulse amplitude: a pulse amplitude of $2.75 \mathrm{~V}$ results in continuous growth, while growth curves for larger voltages are less smooth and feature the well-known drops (Figure $\mathrm{S} 9 \mathrm{k}$ ). A comparison of pre- and post-deposition micrographs of the utilized tip's apex still indicates loss of material, as expected from the growth curves (Figure $\mathbf{S 9}$ a, d). It is still to be shown that $\mathrm{Pt}$ or Pt-20at.\% Ir tips do not degrade with appropriate process parameters. 

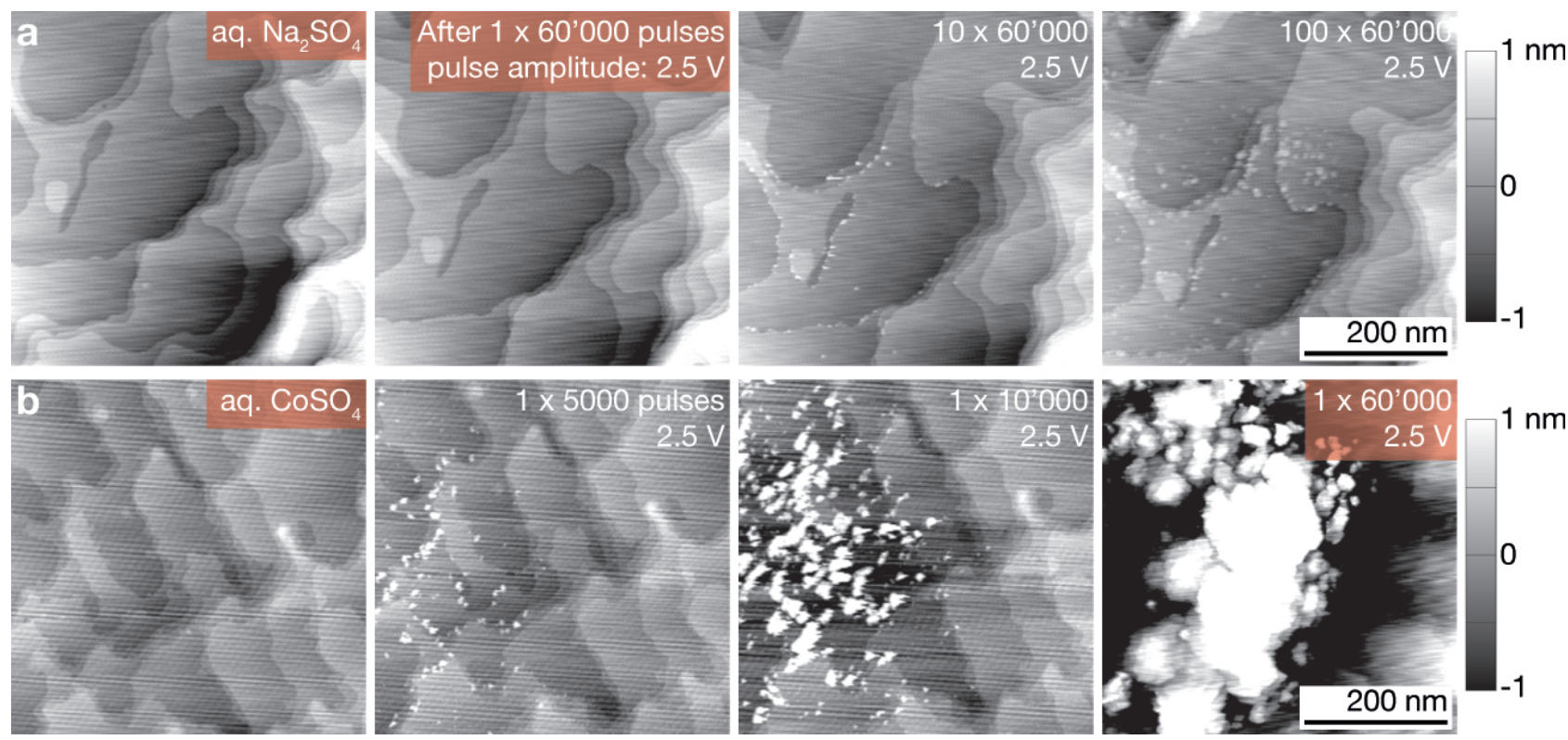

Figure S7: Deposition with Pt tips in aq. $\mathrm{Na}_{2} \mathrm{SO}_{4}$ and $\mathrm{CoSO}_{4}$ solutions. A comparison between deposition experiments in $\mathrm{Co}^{2+}$-free and $\mathrm{Co}^{2+}$-containing electrolytes suggest sufficient stability of $\mathrm{Pt}$ tips for Co deposition: STM scans of a Au surface before and after deposition with stated number of pulses in a, $0.25 \mathrm{M}$ aq. $\mathrm{Na}_{2} \mathrm{SO}_{4}$ and $\mathbf{b}, 0.25 \mathrm{M}$ aq. $\mathrm{CoSO}_{4}$. In the $\mathrm{Co}^{2+}$-containing solution, a significant amount of material is deposited after $1 \times 60000$ pulses. In contrast, no deposition is detected with equal parameters in the $\mathrm{Co}^{2+}$-free solution, and only minor deposition is observed after $100 \times 60000$ pulses. Note: the deposition in the rightmost scan of (b) was initiated on a previously clean surface, while the deposit in the third scan was grown on top of the one in the second scan. Deposition parameters, Pulse width: $20 \mathrm{~ns}$; pulse amplitude: $2.5 \mathrm{~V}$; duty cycle: 0.1 ; dwell distance: $50 \mathrm{~nm}$; $\Phi_{\text {substrate }}$ : $-900 \mathrm{mV}$ (a), $-1000 \mathrm{mV}$ (b); $\Phi_{\text {tip }}:-700 \mathrm{mV}$; tip: Pt. 

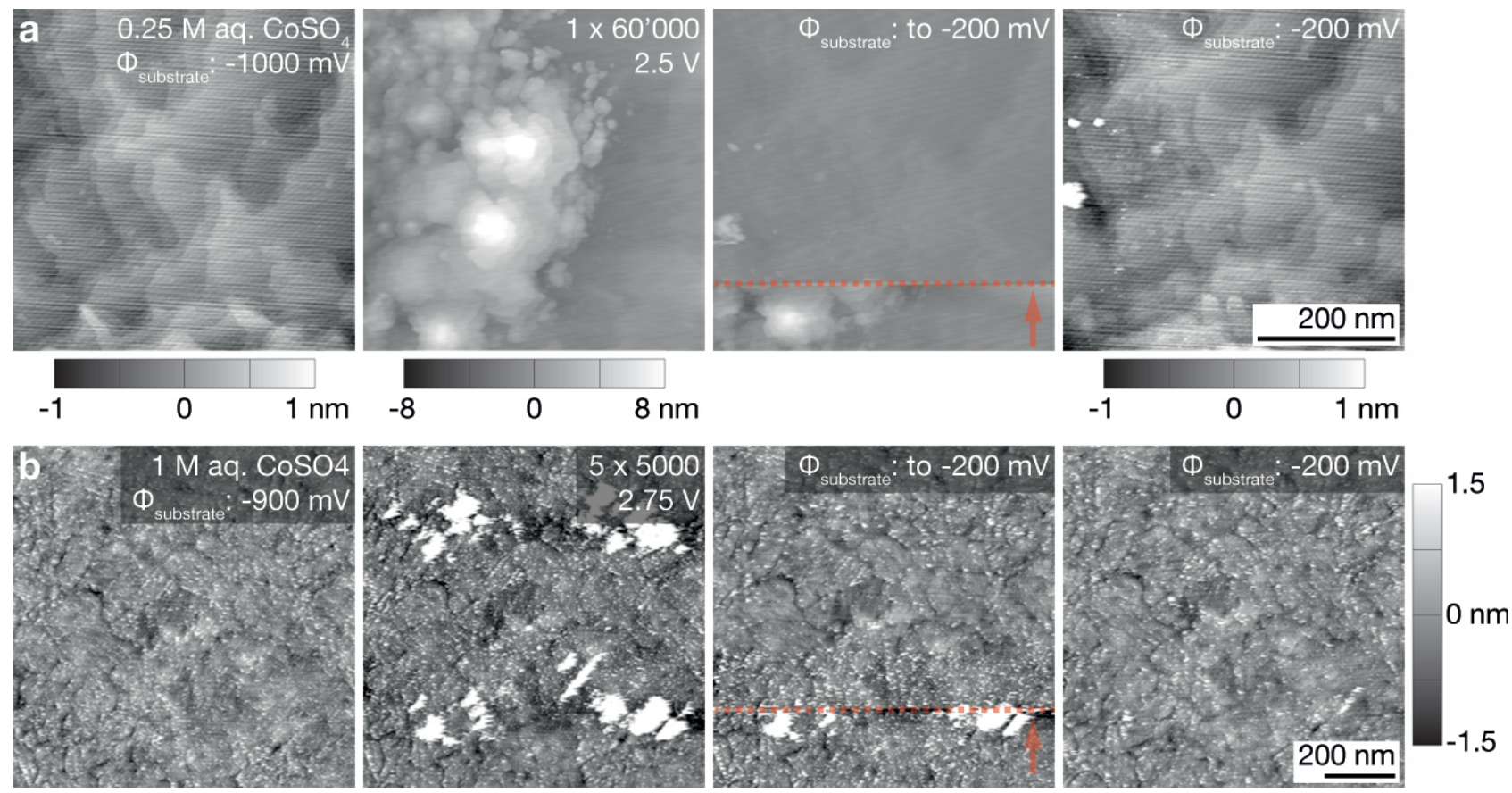

Figure S8: Complete dissolution of deposits. a, STM scans of a Au surface before (first scan) and after deposition in $0.25 \mathrm{M}$ aq. $\mathrm{CoSO}_{4}$ (second scan, same deposit as in Figure S7b). Stepping $\Phi_{\text {substrate }}$ to $-200 \mathrm{mV}$ vs. Au (dashed line in the third scan. The arrow denotes the scan direction) results in the instantaneous dissolution of the deposits. Note the different gray scales of the individual panels. b, Scans of the surface of a Au thin film before and after the deposition of four dots (only $5 \times 5000$ pulses) an after their complete dissolution at $-200 \mathrm{mV}$ vs. Au. Deposition parameters. Pulse width: $20 \mathrm{~ns}$; pulse amplitude: $2.5 \mathrm{~V}$ (a), $2.75 \mathrm{~V}$ (d); duty cycle: 0.1 ; dwell distance: $50 \mathrm{~nm}$; $\Phi_{\text {tip }}$ : $-700 \mathrm{mV}$. tip: Pt (a), Pt-20at.\%Ir (b).
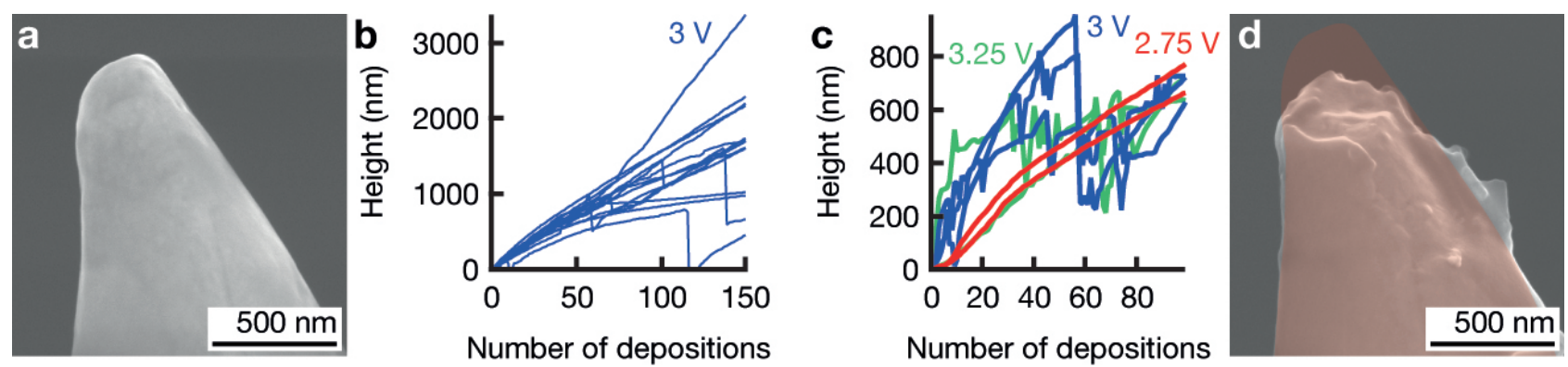

Figure S9: Out-of-plane growth curves with Pt-20at.\% Ir tips. a, d, Pre- and post-experiment SE micrograph of the tip used in (b) and (c). b, Growth curves recorded with a Pt-20at.\%Ir tip. While stable growth up to a height of $2-3 \mu \mathrm{m}$ can be achieved, the growth curves are not free of instabilities. c, Growth curves recorded for pulse amplitudes of 2.75, 3 and $3.25 \mathrm{~V}$. Low amplitudes enable continuous growth, while larger voltages cause growth instabilities. Deposition parameters. Electrolyte: $1 \mathrm{M}$ aq. $\mathrm{CoSO}_{4}$; pulse width: $20 \mathrm{~ns}$; pulse amplitude: $3 \mathrm{~V}$ (a), 2.75-3.25 V (b); duty cycle: 0.1; pulses per deposition event: 30000 (a), $10000-30000$ (b); dwell distance: $50 \mathrm{~nm}$; dwell time: 0.5 s; tip: Pt-20at.\%Ir. 